JOURNAL OF

FUNCTION SPACES AND APPLICATIONS

Volume 8, Number 2 (2010), 129-165 (c) 2010, Scientific Horizon

http://www.jfsa.net

\title{
Atomic, molecular and wavelet decomposition of generalized 2-microlocal Besov spaces
}

\author{
Henning Kempka
}

(Communicated by Hans Triebel)

2000 Mathematics Subject Classification. 42B35, 42B20, 46E35, 47G30.

Keywords and phrases. 2-microlocal spaces, wavelet decomposition, weighted Besov spaces.

\footnotetext{
Abstract. We introduce generalized 2-microlocal Besov spaces and give characterizations in decomposition spaces by atoms, molecules and wavelets. We apply the wavelet decomposition to prove that the 2-microlocal spaces are invariant under the action of pseudodifferential operators of order 0 .
}

\section{Introduction}

The concept of 2-microlocal analysis or 2-microlocal function spaces is due to J.M. Bony (see [2]). It is an appropriate instrument to describe the local regularity and the oscillatory behavior of functions near singularities.

The approach is Fourier-analytical using Littlewood-Paley-analysis of distributions. The theory has been elaborated and widely used in fractal analysis and signal processing by several authors. We refer to S. Jaffard ([8], $[9])$, Y. Meyer ([14]) and J. Lévy Véhel \& S. Seuret ([12]). These works have been generalized in different directions by P. Andersson ([1]), H. Xu([15], [23]), and S. Moritoh \& T. Yamada ([16]).

The main achievements are closely related to the use of wavelet methods and, as a consequence, to wavelet characterizations of 2-microlocal spaces. Here, we intend to give a unified Fourier-analytical approach to generalized 
2-microlocal Besov spaces and we are interested in characterizations by various decompositions.

The paper is organized as follows. After recalling some preliminaries and notation in Section 2 we define the concept of 2-microlocal Besov spaces and repeat some results from [10] in Section 3.

In the main Sections 4 and 5 we present the characterizations of the 2-microlocal Besov spaces by decomposition in atoms, molecules and in wavelets. Only the proofs of the main theorems can be found in these sections, whereas the proofs of the more technical lemmas are shifted to the Appendix.

Finally, in Section 6 we show that the 2-microlocal Besov spaces are invariant under the action of certain Calderón-Zygmund operators introduced in [13] and the action of pseudodifferential operators of order 0 .

\section{Preliminaries}

As usual, $\mathbb{R}^{n}$ denotes the $n$-dimensional Euclidean space, $\mathbb{N}$ is the collection of all natural numbers and $\mathbb{N}_{0}=\mathbb{N} \cup\{0\}, \mathbb{Z}$ and $\mathbb{C}$ stand for the sets of integers and complex numbers, respectively.

The points of the Euclidian space $x \in \mathbb{R}^{n}$ are denoted by $x=$ $\left(x_{1}, \ldots, x_{n}\right)$. If $\beta=\left(\beta_{1}, \ldots, \beta_{n}\right) \in \mathbb{N}_{0}^{n}$ is a multi-index, then its length is denoted by $|\beta|=\sum_{j=1}^{n} \beta_{j}$. The derivatives $D^{\beta}=\partial^{|\beta|} / \partial_{1}^{\beta_{1}} \cdots \partial_{n}^{\beta_{n}}$ have to be understood in the distributional sense. We put $x^{\beta}=x_{1}^{\beta_{1}} \cdots x_{n}^{\beta_{n}}$.

The Schwartz space $\mathcal{S}\left(\mathbb{R}^{n}\right)$ is the space of all complex valued rapidly decreasing and infinitely differentiable functions on $\mathbb{R}^{n}$. Its topology is generated by the norms

$$
\|\varphi\|_{k, l}=\sup _{x \in \mathbb{R}^{n}}(1+|x|)^{k} \sum_{|\beta| \leq l}\left|D^{\beta} \varphi(x)\right|, \quad k, l \in \mathbb{N}_{0} .
$$

A linear mapping $f: \mathcal{S}\left(\mathbb{R}^{n}\right) \rightarrow \mathbb{C}$ is called a tempered distribution, if there exists a constant $c>0$ and $k, l \in \mathbb{N}_{0}$ such that $|f(\varphi)| \leq c\|\varphi\|_{k, l}$ holds for all $\varphi \in \mathcal{S}\left(\mathbb{R}^{n}\right)$. The collection of all such mappings is denoted by $\mathcal{S}^{\prime}\left(\mathbb{R}^{n}\right)$. The Fourier transform is defined on both spaces $\mathcal{S}\left(\mathbb{R}^{n}\right)$ and $\mathcal{S}^{\prime}\left(\mathbb{R}^{n}\right)$ and is given by

$$
(\mathcal{F} f)(\varphi):=f(\mathcal{F} \varphi), \quad \varphi \in \mathcal{S}\left(\mathbb{R}^{n}\right), \quad f \in \mathcal{S}^{\prime}\left(\mathbb{R}^{n}\right)
$$


where

$$
\mathcal{F} \varphi(\xi):=\frac{1}{(2 \pi)^{n / 2}} \int_{\mathbb{R}^{n}} \mathrm{e}^{-i x \cdot \xi} \varphi(x) d x .
$$

Here $x \cdot \xi=x_{1} \xi_{1}+\cdots+x_{n} \xi_{n}$ stands for the inner product. The inverse Fourier transform is denoted by $\mathcal{F}^{-1} \varphi$ or $\varphi^{\vee}$ and we often write $\hat{\varphi}$ instead of $\mathcal{F} \varphi$.

We say that a vector space $E$ is a quasi-Banach space, if it is complete and quasi-normed by $\|\cdot \mid E\|$. That means $\|\cdot \mid E\|$ fulfills the norm conditions but the triangle inequality changes to

$$
\|x+y|E\|\leq c\| x| E\|+c\|y \mid E\|
$$

for $c \geq 1$. If $c=1$ in (1) then $\|\cdot \mid E\|$ is a norm and $E$ is called a Banach space. As usual $L_{p}\left(\mathbb{R}^{n}\right)$ for $0<p \leq \infty$ stands for the Lebesgue spaces on $\mathbb{R}^{n}$ normed by (quasi-normed if $p<1$ )

$$
\begin{aligned}
\left\|f \mid L_{p}\left(\mathbb{R}^{n}\right)\right\| & =\left(\int_{\mathbb{R}^{n}}|f(x)|^{p} d x\right)^{1 / p} \quad \text { for } 0<p<\infty \text { and } \\
\left\|f \mid L_{\infty}\left(\mathbb{R}^{n}\right)\right\| & =\underset{x \in \mathbb{R}^{n}}{\operatorname{ess-sup}}|f(x)| .
\end{aligned}
$$

Let $w$ be a positive measurable function. We define the weighted Lebesgue space $L_{p}\left(\mathbb{R}^{n}, w\right)$ by $\left\|f\left|L_{p}\left(\mathbb{R}^{n}, w\right)\|=\| w f\right| L_{p}\left(\mathbb{R}^{n}\right)\right\|$.

For a sequence $f=\left\{f_{j}\right\}_{j=0}^{\infty}$ of vectors $f_{j} \in E$ the sequence spaces $\ell_{q}(E)$ for $0<q \leq \infty$ are normed by (quasi-normed if $q<1$ or $E$ is a quasi-Banach space)

$$
\begin{aligned}
\left\|f \mid \ell_{q}(E)\right\| & =\left(\sum_{j=0}^{\infty}\left\|f_{j} \mid E\right\|^{q}\right)^{1 / q} \quad \text { for } 0<q<\infty \text { and } \\
\left\|f \mid \ell_{\infty}(E)\right\| & =\sup _{j \in \mathbb{N}_{0}}\left\|f_{j} \mid E\right\| .
\end{aligned}
$$

If $E=\mathbb{C}$ then we shortly denote $\ell_{q}(\mathbb{C})$ by $\ell_{q}$.

The constant $c$ stands for all unimportant constants. So the value of the constant $c$ may change from one occurrence to another. By $a_{k} \sim b_{k}$ we mean that there are two constants $c_{1}, c_{2}>0$ such that $c_{1} a_{k} \leq b_{k} \leq c_{2} a_{k}$ for all admissible $k$. 


\section{Definitions and basic properties}

In this section we present the Fourier-analytical definition of generalized 2-microlocal Besov spaces $B_{p q}^{s, m l o c}\left(\mathbb{R}^{n}, \boldsymbol{w}\right)$ and we prove the basic properties in analogy to the classical Besov spaces. To this end we need smooth resolutions of unity and we introduce admissible weight sequences.

Definition 1 (Admissible weight sequence). Let $\alpha, \alpha_{1}, \alpha_{2} \geq 0$. A sequence of non-negative measurable functions $\boldsymbol{w}=\left\{w_{j}\right\}_{j=0}^{\infty}$ belongs to the class $\mathcal{W}_{\alpha_{1}, \alpha_{2}}^{\alpha}$ if and only if

(i) There exists a constant $\mathrm{C}>0$ such that

$0<w_{j}(x) \leq \mathrm{C} w_{j}(y)\left(1+2^{j}|x-y|\right)^{\alpha} \quad$ for all $j \in \mathbb{N}_{0}$ and all $x, y \in \mathbb{R}^{n}$

(ii) For all $j \in \mathbb{N}_{0}$ we have

$$
2^{-\alpha_{1}} w_{j}(x) \leq w_{j+1}(x) \leq 2^{\alpha_{2}} w_{j}(x) \text { for all } x \in \mathbb{R}^{n} \text {. }
$$

Such a system $\left\{w_{j}\right\}_{j=0}^{\infty} \in \mathcal{W}_{\alpha_{1}, \alpha_{2}}^{\alpha}$ is called admissible weight sequence.

A non-negative measurable function $\varrho$ is called an admissible weight function if there exist constants $\alpha_{\varrho} \geq 0$ and $\mathrm{C}_{\varrho}>0$, such that

$$
0<\varrho(x) \leq \mathrm{C}_{\varrho} \varrho(y)(1+|x-y|)^{\alpha_{\varrho}} \text { holds for every } x, y \in \mathbb{R}^{n} .
$$

If $\boldsymbol{w}=\left\{w_{j}\right\}_{j=0}^{\infty}$ is an admissible weight sequence, each $w_{j}$ is an admissible weight function, but in general the constant $\mathrm{C}_{w_{j}}$ depends on $j \in \mathbb{N}_{0}$. If we use $\boldsymbol{w} \in \mathcal{W}_{\alpha_{1}, \alpha_{2}}^{\alpha}$ without any restrictions, then $\alpha, \alpha_{1}, \alpha_{2} \geq 0$ are arbitrary but fixed numbers.

A fundamental example of an admissible weight sequence is given by the 2-microlocal weights. For a fixed nonempty set $U \subset \mathbb{R}^{n}$ and $s^{\prime} \in \mathbb{R}$ they are given by

$$
w_{j}(x):=\left(1+2^{j} \operatorname{dist}(x, U)\right)^{s^{\prime}}
$$

where $\operatorname{dist}(x, U)=\inf _{z \in U}|x-z|$ is the distance of $x \in \mathbb{R}^{n}$ from $U$. A special case is $U=\left\{x_{0}\right\}$ for $x_{0} \in \mathbb{R}^{n}$. Then $\operatorname{dist}(U, x)=\left|x-x_{0}\right|$ and we get the well known 2-microlocal weights [9] treated by many authors

$$
w_{j}(x)=\left(1+2^{j}\left|x-x_{0}\right|\right)^{s^{\prime}} \quad \text { for } j \in \mathbb{N}_{0} .
$$

If $U$ is an open subset of $\mathbb{R}^{n}$, we get the weight sequence Moritoh and Yamada used in [16]. Another example of an admissible weight sequence is 
given for fixed $s^{\prime} \in \mathbb{R}$ by

$$
w_{j}(x)=\left(1+2^{j} w(x)\right)^{s^{\prime} / \beta},
$$

where $w: \mathbb{R}^{n} \rightarrow[0, \infty)$ is a measurable function with the following properties: There are constants $\mathcal{C}_{1}, \mathcal{C}_{2} \geq 1$ and $\beta \geq 1$ such that for all $x, y \in \mathbb{R}^{n}$

$$
0 \leq w(x) \leq \mathcal{C}_{1} w(y)+\mathcal{C}_{2}|x-y|^{\beta} .
$$

As a special case we choose $w: \mathbb{R}^{n} \rightarrow[0, \infty)$ subadditiv, that is

$$
\begin{aligned}
0 \leq w(x+y) & \leq \tilde{c}_{1}(w(x)+w(y)) \quad \text { and in addition we need } \\
w(x) & \leq \tilde{c}_{2}|x|^{\beta} \quad \text { for all } x, y \in \mathbb{R}^{n} \text { and fixed } \tilde{c}_{1}, \tilde{c}_{2}, \beta \geq 1 .
\end{aligned}
$$

Thus we have (6) with $\mathcal{C}_{1}=\tilde{c}_{1}$ and $\mathcal{C}_{2}=\tilde{c}_{1} \tilde{c}_{2}$ and we can define the admissible weight sequence as in (5). For all examples of admissible weight sequences, considered above, it is easy to show, that $\boldsymbol{w} \in \mathcal{W}_{\alpha_{1}, \alpha_{2}}^{\alpha}$ if $\left|s^{\prime}\right| \leq \alpha$, $\max \left(0,-s^{\prime}\right) \leq \alpha_{1}$ and $\max \left(0, s^{\prime}\right) \leq \alpha_{2}$.

Next we define the resolution of unity.

Definition 2 (Resolution of unity). A system $\varphi=\left\{\varphi_{j}\right\}_{j=0}^{\infty} \subset \mathcal{S}\left(\mathbb{R}^{n}\right)$ belongs to the class $\Phi\left(\mathbb{R}^{n}\right)$ if and only if

(i)

$$
\begin{aligned}
& \operatorname{supp} \varphi_{0} \subseteq\left\{x \in \mathbb{R}^{n}:|x| \leq 2\right\} \quad \text { and for all } j \in \mathbb{N} \\
& \operatorname{supp} \varphi_{j} \subseteq\left\{x \in \mathbb{R}^{n}: 2^{j-1} \leq|x| \leq 2^{j+1}\right\} .
\end{aligned}
$$

(ii) For each $\beta \in \mathbb{N}_{0}^{n}$ there exist constants $c_{\beta}>0$ such that

$$
2^{j|\beta|} \sup _{x \in \mathbb{R}^{n}}\left|D^{\beta} \varphi_{j}(x)\right| \leq c_{\beta} \quad \text { holds for all } j \in \mathbb{N}_{0} .
$$

(iii) For all $x \in \mathbb{R}^{n}$ we have

$$
\sum_{j=0}^{\infty} \varphi_{j}(x)=1 .
$$

Remark 1. Such a resolution of unity can easily be constructed. Consider the following example. Let $\varphi_{0} \in \mathcal{S}\left(\mathbb{R}^{n}\right)$ with $\varphi_{0}(x)=1$ for $|x| \leq 1$ and $\operatorname{supp} \varphi_{0} \subseteq\left\{x \in \mathbb{R}^{n}:|x| \leq 2\right\}$. For $j \geq 1$ we define

$$
\varphi(x)=\varphi_{0}(x)-\varphi_{0}(2 x) \quad \text { and } \quad \varphi_{j}(x)=\varphi\left(2^{-j} x\right) .
$$


Now it is obvious that $\varphi=\left\{\varphi_{j}\right\}_{j \in \mathbb{N}_{0}} \in \Phi\left(\mathbb{R}^{n}\right)$.

Definition 3. Let $\left\{\varphi_{j}\right\}_{j \in \mathbb{N}_{0}} \in \Phi\left(\mathbb{R}^{n}\right)$ be a resolution of unity and let $\boldsymbol{w}=\left\{w_{j}\right\}_{j \in \mathbb{N}_{0}} \in \mathcal{W}_{\alpha_{1}, \alpha_{2}}^{\alpha}$. Further, let $0<p \leq \infty, 0<q \leq \infty$ and $s \in \mathbb{R}$. Then we define

$$
\begin{gathered}
B_{p q}^{s, m l o c}\left(\mathbb{R}^{n}, \boldsymbol{w}\right)=\left\{f \in \mathcal{S}^{\prime}\left(\mathbb{R}^{n}\right):\left\|f \mid B_{p q}^{s, m l o c}\left(\mathbb{R}^{n}, \boldsymbol{w}\right)\right\|_{\varphi}<\infty\right\} \quad, \text { where } \\
\left\|f \mid B_{p q}^{s, m l o c}\left(\mathbb{R}^{n}, \boldsymbol{w}\right)\right\|_{\varphi}=\left(\sum_{j=0}^{\infty} 2^{j s q}\left\|w_{j}\left(\varphi_{j} \hat{f}\right)^{\vee} \mid L_{p}\left(\mathbb{R}^{n}\right)\right\|^{q}\right)^{1 / q}
\end{gathered}
$$

with the usual modifications if $q$ is equal to infinity.

One recognizes immediately that for $w_{j}(x)=1$ for all $x \in \mathbb{R}^{n}$ and all $j \in \mathbb{N}_{0}$ we get the usual Besov spaces, see [18]. If one defines the admissible weight sequence as $w_{j}(x)=\varrho(x)$ for each $j \in \mathbb{N}_{0}$ and $\varrho$ being an admissible weight satisfying (2), we obtain weighted Besov spaces, see [4, Chapter 4].

Applying a Fourier multiplier theorem for weighted Lebesgue spaces of entire analytic functions as in Subsection 1.7.5 in [17] we can prove that Definition 3 is independent of the chosen resolution of unity $\left\{\varphi_{j}\right\}_{j \in \mathbb{N}_{0}} \in$ $\Phi\left(\mathbb{R}^{n}\right)$, in the sense of equivalent quasi-norms. To this end, we can suppress the index $\varphi$ in the notation of the norm.

Moreover, as in $\left[18\right.$, Subsection 2.3.3] we can prove that $B_{p q}^{s, m l o c}\left(\mathbb{R}^{n}, \boldsymbol{w}\right)$ is a quasi-Banach space for all $s \in \mathbb{R}$ and $0<p, q \leq \infty$ and even a Banach space in the case $p, q \geq 1$. Also, we have for all admissible weight sequences and all $s, p$ and $q$

$$
\mathcal{S}\left(\mathbb{R}^{n}\right) \subset B_{p q}^{s, m l o c}\left(\mathbb{R}^{n}, \boldsymbol{w}\right) \subset \mathcal{S}^{\prime}\left(\mathbb{R}^{n}\right),
$$

where $\mathcal{S}\left(\mathbb{R}^{n}\right)$ is dense in $B_{p q}^{s, m l o c}\left(\mathbb{R}^{n}, \boldsymbol{w}\right)$ for $0<p, q<\infty$. See [10] for details. Now, let us recall some results from [10] we need later on.

Proposition 1. Let $s \in \mathbb{R}, \boldsymbol{w}=\left\{w_{j}\right\}_{j \in \mathbb{N}_{0}} \in \mathcal{W}_{\alpha_{1}, \alpha_{2}}^{\alpha}, 0<p, q \leq \infty$ and let $m \in \mathbb{N}_{0}$. Then

$$
\sum_{|\beta| \leq m}\left\|D^{\beta} f \mid B_{p q}^{s-m, m l o c}\left(\mathbb{R}^{n}, \boldsymbol{w}\right)\right\| \text { and }\left\|f \mid B_{p q}^{s, m l o c}\left(\mathbb{R}^{n}, \boldsymbol{w}\right)\right\|
$$

are equivalent quasi-norms on $B_{p q}^{s, m l o c}\left(\mathbb{R}^{n}, \boldsymbol{w}\right)$.

The spaces $B_{p q}^{s, m l o c}\left(\mathbb{R}^{n}, \boldsymbol{w}\right)$ have the lift property. To be precise, let us define the lift operator $I_{\sigma}$ by

$$
I_{\sigma}: f \mapsto\left(\langle\xi\rangle^{\sigma} \hat{f}\right)^{\vee}
$$


where $\langle\xi\rangle=\left(1+|\xi|^{2}\right)^{1 / 2}$ and $z \in \mathbb{R}$.

Proposition 2. Let $s, \sigma \in \mathbb{R}$ and $\boldsymbol{w}=\left\{w_{j}\right\}_{j \in \mathbb{N}_{0}} \in \mathcal{W}_{\alpha_{1}, \alpha_{2}}^{\alpha}$. Moreover, let $0<p \leq \infty$ and $0<q \leq \infty$. Then $I_{\sigma}$ maps $B_{p q}^{s, m l o c}\left(\mathbb{R}^{n}, \boldsymbol{w}\right)$ isomorphically onto $B_{p q}^{s-\sigma, m l o c}\left(\mathbb{R}^{n}, \boldsymbol{w}\right)$. Further, $\left\|I_{\sigma} f \mid B_{p q}^{s-\sigma, m l o c}\left(\mathbb{R}^{n}, \boldsymbol{w}\right)\right\|$ is an equivalent quasi-norm on $B_{p q}^{s, m l o c}\left(\mathbb{R}^{n}, \boldsymbol{w}\right)$.

The next result is the characterization of $B_{p q}^{s, m l o c}\left(\mathbb{R}^{n}, \boldsymbol{w}\right)$ by local means. Therefore, we define for $\left\{\psi_{k}\right\} \in \mathcal{S}\left(\mathbb{R}^{n}\right), f \in \mathcal{S}^{\prime}\left(\mathbb{R}^{n}\right)$ and $a>0$ the Peetre maximal operator by

$$
\left(\psi_{k}^{*} f\right)_{a}(x)=\sup _{y \in \mathbb{R}^{n}} \frac{\left|\left(\psi_{k} * f\right)(y)\right|}{1+\left|2^{k}(y-x)\right|^{a}} \quad\left(k \in \mathbb{N}_{0}, x \in \mathbb{R}^{n}\right) .
$$

Proposition 3. Let $\boldsymbol{w}=\left\{w_{k}\right\}_{k \in \mathbb{N}_{0}} \in \mathcal{W}_{\alpha_{1}, \alpha_{2}}^{\alpha}, 0<p, q \leq \infty$ and let $s, a \in \mathbb{R}, R \in \mathbb{N}_{0}$ with $a>\frac{n}{p}+\alpha$ and $R>s+\alpha_{2}$. Let $\psi_{0}, \psi_{1} \in \mathcal{S}\left(\mathbb{R}^{n}\right)$ such that

$$
D^{\beta} \psi_{1}^{\vee}(0)=0, \quad \text { for } 0 \leq|\beta|<R,
$$

and

$$
\begin{array}{lll}
\left|\psi_{0}^{\vee}(x)\right|>0 & \text { on } & \left\{x \in \mathbb{R}^{n}:|x|<\varepsilon\right\} \\
\left|\psi_{1}^{\vee}(x)\right|>0 & \text { on } & \left\{x \in \mathbb{R}^{n}: \varepsilon / 2<|x|<2 \varepsilon\right\}
\end{array}
$$

for some $\varepsilon>0$. Further, let $\psi_{k}(x)=2^{(k-1) n} \psi_{1}\left(2^{k-1} x\right)$, then

$$
\left\|f\left|B_{p q}^{s, m l o c}\left(\mathbb{R}^{n}, \boldsymbol{w}\right)\|\sim\| 2^{k s}\left(\psi_{k} * f\right) w_{k}\right| l_{q}\left(L_{p}\right)\right\| \sim\left\|2^{k s}\left(\psi_{k}^{*} f\right)_{a} w_{k} \mid l_{q}\left(L_{p}\right)\right\|
$$

holds for all $f \in \mathcal{S}^{\prime}\left(\mathbb{R}^{n}\right)$.

If $R=0$, then we do not need any moment conditions (9) on $\psi_{1}$. Now, we present an elementary embedding in the scale of $B_{p q}^{s, m l o c}\left(\mathbb{R}^{n}, \boldsymbol{w}\right)$.

Proposition 4. Let $s \in \mathbb{R}$ and $\boldsymbol{w}, \varrho \in \mathcal{W}_{\alpha_{1}, \alpha_{2}}^{\alpha}$ with $\frac{w_{j}(x)}{\varrho_{j}(x)} \leq c$ for all $j \in \mathbb{N}_{0}$ and $x \in \mathbb{R}^{n}$. If $0<p \leq \infty, 0<q_{1} \leq \infty, 0<q_{2} \leq \infty$ and $\varepsilon>0$, then

$$
B_{p q_{1}}^{s, m l o c}\left(\mathbb{R}^{n}, \varrho\right) \hookrightarrow B_{p q_{2}}^{s-\varepsilon, m l o c}\left(\mathbb{R}^{n}, \boldsymbol{w}\right) .
$$

For further embeddings in the general scale of $B_{p q}^{s, m l o c}\left(\mathbb{R}^{n}, \boldsymbol{w}\right)$ and also in the special scale $B_{p q}^{s, s^{\prime}}\left(\mathbb{R}^{n}, U\right)$ with the 2-microlocal weights $w_{j}(x)=$ $\left(1+2^{j} \operatorname{dist}(x, U)\right)^{s^{\prime}}$, we refer to [10]. 


\section{Decomposition by atoms and molecules}

In this section, we present two decomposition theorems. We characterize the spaces $B_{p q}^{s, m l o c}\left(\mathbb{R}^{n}, \boldsymbol{w}\right)$ via decompositions by atoms and molecules. First we introduce the basic notation.

4.1 Sequence Spaces. First of all, we define for $\nu \in \mathbb{N}_{0}$ and $m \in \mathbb{Z}^{n}$ the closed cube $Q_{\nu m}$ with center in $2^{-\nu} m$ and with sides parallel to the axes and length $2^{-\nu}$. By $\chi_{\nu m}$ we denote the characteristic function of the cube $Q_{\nu m}$, defined by

$$
\chi_{\nu m}(x)=\chi_{Q_{\nu m}}(x)= \begin{cases}1, & \text { if } x \in Q_{\nu m} \\ 0, & \text { if } x \notin Q_{\nu m}\end{cases}
$$

Definition 4. Let $\boldsymbol{w}=\left\{w_{k}\right\}_{k \in \mathbb{N}_{0}} \in \mathcal{W}_{\alpha_{1}, \alpha_{2}}^{\alpha}, s \in \mathbb{R}$ and let $0<p, q \leq \infty$.

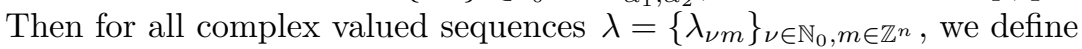

$$
b_{p q}^{s, m l o c}(\boldsymbol{w})=\left\{\lambda \subset \mathbb{C}\left\|\lambda \mid b_{p q}^{s, m l o c}(\boldsymbol{w})\right\|<\infty\right\}
$$

where

$$
\left\|\lambda \mid b_{p q}^{s, m l o c}(\boldsymbol{w})\right\|:=\left(\sum_{\nu=0}^{\infty} 2^{\nu\left(s-\frac{n}{p}\right) q}\left(\sum_{m \in \mathbb{Z}^{n}} w_{\nu}^{p}\left(2^{-\nu} m\right)\left|\lambda_{\nu m}\right|^{p}\right)^{q / p}\right)^{1 / q}
$$

with the usual modifications if $p$ or $q$ are equal to infinity.

Remark 2. Observe, that

$$
\left\|\lambda \mid b_{p q}^{s, m l o c}(\boldsymbol{w})\right\| \sim\left(\sum_{\nu=0}^{\infty} 2^{\nu s q}\left\|\sum_{m \in \mathbb{Z}^{n}} \lambda_{\nu m} \chi_{\nu m}(\cdot) w_{\nu}(\cdot) \mid L_{p}\left(\mathbb{R}^{n}\right)\right\|^{q}\right)^{1 / q}
$$

4.2 Atoms and Molecules. Atoms are the building blocks for atomic decompositions.

Definition 5. Let $K, L \in \mathbb{N}_{0}$ and let $\gamma>1$. A $K$-times continuous differentiable function $a \in C^{K}\left(\mathbb{R}^{n}\right)$ is called $[K, L]$-atom centered at $Q_{\nu m}$, $\nu \in \mathbb{N}_{0}$ and $m \in \mathbb{Z}^{n}$, if

$$
\begin{aligned}
& \operatorname{supp} a \subseteq \gamma Q_{\nu m}, \\
&\left|D^{\beta} a(x)\right| \leq 2^{|\beta| \nu}, \quad \text { for } 0 \leq|\beta| \leq K
\end{aligned}
$$


and if

$$
\int_{R^{n}} x^{\beta} a(x) d x=0 \quad \text { for } \quad 0 \leq|\beta|<L \text { and } \nu \geq 1 .
$$

If an atom $a$ is centered at $Q_{\nu m}$, that means if it fulfills (13), then we denote it by $a_{\nu m}$. We recall the definition $x^{\beta}=x_{1}^{\beta_{1}} \cdots x_{n}^{\beta_{n}}$ and point out that for $\nu=0$ or $L=0$ there are no moment conditions (15) required.

Definition 6. Let $K, L \in \mathbb{N}_{0}$ and let $M>0$. A $K$-times continuous differentiable function $\mu \in C^{K}\left(\mathbb{R}^{n}\right)$ is called $[K, L, M]$-molecule concentrated in $Q_{\nu m}$, if for some $\nu \in \mathbb{N}_{0}$ and $m \in \mathbb{Z}^{n}$

$$
\left|D^{\beta} \mu(x)\right| \leq 2^{|\beta| \nu}\left(1+2^{\nu}\left|x-2^{-\nu} m\right|\right)^{-M}, \quad \text { for } 0 \leq|\beta| \leq K
$$

and

$$
\int_{R^{n}} x^{\beta} \mu(x) d x=0 \quad \text { if } \quad 0 \leq|\beta|<L \text { and } \nu \geq 1 .
$$

Remark 3. (a) For $L=0$ or $\nu=0$ there are no moment conditions (17) required. If a molecule is concentrated in $Q_{\nu m}$, that means it satisfies (16), then it is denoted by $\mu_{\nu m}$.

(b) If $a_{\nu m}$ is a $[K, L]$-atom then it is a $[K, L, M]$-molecule for every $M>0$.

First, we show the convergence of the molecular decomposition. To this end, we introduce the number $\sigma_{p}$, defined by $\sigma_{p}=\max (0, n(1 / p-1))$. Remember that the proofs of the lemmas can be found in the Appendix.

Lemma 1. Let $\boldsymbol{w}=\left\{w_{k}\right\}_{k \in \mathbb{N}_{0}} \in \mathcal{W}_{\alpha_{1}, \alpha_{2}}^{\alpha}$ and let $0<p, q \leq \infty, s \in \mathbb{R}$. Furthermore, let $K, L \in \mathbb{N}_{0}$ and $M>0$ with

$$
L>\sigma_{p}-s+\alpha_{1}, \quad K \in \mathbb{N}_{0} \text { and } M \text { large enough } .
$$

If $\lambda \in b_{p q}^{s, m l o c}(\boldsymbol{w})$ and $\left\{\mu_{\nu m}\right\}_{\nu \mathbb{N}_{0}, m \in \mathbb{Z}^{n}}$ are $[K, L, M]$-molecules concentrated in $Q_{\nu m}$, then the sum

$$
\sum_{\nu=0}^{\infty} \sum_{m \in \mathbb{Z}^{n}} \lambda_{\nu m} \mu_{\nu m}(x)
$$

converges in $\mathcal{S}^{\prime}\left(\mathbb{R}^{n}\right)$.

Remark 4. A sufficient condition on $M$ is $M>L+2 \alpha+2 n$.

We also need a representation formula of Calderón type. The proof can be found in [5, Theorem 2.6]. 
Lemma 2. Let $\left\{\varphi_{j}\right\}_{j \in \mathbb{N}_{0}} \in \Phi\left(\mathbb{R}^{n}\right)$ be a resolution of unity and let $M \in \mathbb{N}$. Then there exist functions $\theta_{0}, \theta \in \mathcal{S}\left(\mathbb{R}^{n}\right)$ with:

$$
\begin{gathered}
\operatorname{supp} \theta_{0}, \operatorname{supp} \theta \subseteq\left\{x \in \mathbb{R}^{n}:|x| \leq 1\right\}, \\
\left|\hat{\theta}_{0}(\xi)\right| \geq c_{0}>0 \quad \text { for } \quad|\xi| \leq 2, \\
|\hat{\theta}(\xi)| \geq c>0 \quad \text { for } \quad \frac{1}{2} \leq|\xi| \leq 2 \\
\int_{\mathbb{R}^{n}} x^{\gamma} \theta(x) d x=0 \quad \text { for } \quad 0 \leq|\gamma| \leq M
\end{gathered}
$$

such that

$$
\hat{\theta}_{0}(\xi) \hat{\psi}_{0}(\xi)+\sum_{j=1}^{\infty} \hat{\theta}\left(2^{-j} \xi\right) \hat{\psi}\left(2^{-j} \xi\right)=1 \quad \text {, for all } \xi \in \mathbb{R}^{n}
$$

where the functions $\psi_{0}, \psi \in \mathcal{S}\left(\mathbb{R}^{n}\right)$ are defined by

$$
\hat{\psi}_{0}(\xi)=\frac{\varphi_{0}(\xi)}{\hat{\theta}_{0}(\xi)} \quad \text { and } \quad \hat{\psi}(\xi)=\frac{\varphi_{1}(2 \xi)}{\hat{\theta}(\xi)} .
$$

We have already seen that the sum in (18) converges in $\mathcal{S}^{\prime}\left(\mathbb{R}^{n}\right)$ under the conditions of Lemma 1. Now we come to the atomic decomposition theorem.

Theorem 1. Let $\boldsymbol{w}=\left\{w_{k}\right\}_{k \in \mathbb{N}_{0}} \in \mathcal{W}_{\alpha_{1}, \alpha_{2}}^{\alpha}, s \in \mathbb{R}$ and let $0<p, q \leq \infty$. Furthermore, let $K, L \in \mathbb{N}_{0}$ with

$$
K>s+\alpha_{2} \quad \text { and } \quad L>\sigma_{p}-s+\alpha_{1} .
$$

For each $f \in B_{p q}^{s, m l o c}\left(\mathbb{R}^{n}, \boldsymbol{w}\right)$ there exist $\lambda \in b_{p q}^{s, m l o c}(\boldsymbol{w})$ and $[K, L]$-atoms $\left\{a_{\nu m}\right\}$ centered at $Q_{\nu m}$ such that the representation

$$
f=\sum_{\nu=0}^{\infty} \sum_{m \in \mathbb{Z}^{n}} \lambda_{\nu m} a_{\nu m}, \quad \text { convergence being in } \mathcal{S}^{\prime}\left(\mathbb{R}^{n}\right),
$$

holds. Moreover,

$$
\left\|\lambda\left|b_{p q}^{s, m l o c}(\boldsymbol{w})\|\leq c\| f\right| B_{p q}^{s, m l o c}\left(\mathbb{R}^{n}, \boldsymbol{w}\right)\right\|,
$$

where the constant $c$ is universal for all $f \in B_{p q}^{s, m l o c}\left(\mathbb{R}^{n}, \boldsymbol{w}\right)$.

Proof. Since $[K, L]$-atoms are $[K, L, M]$-molecules for every $M>0$ the convergence in $\mathcal{S}^{\prime}\left(\mathbb{R}^{n}\right)$ has already been stated in Lemma 1. 
The proof relies on the method used in the proof of [7, Theorem 5.11]. We use Lemma 2 with $M=L-1$, the functions $\theta_{0}, \theta \in \mathcal{S}\left(\mathbb{R}^{n}\right)$ with the properties (19)-(23) and the functions $\psi_{0}, \psi \in \mathcal{S}\left(\mathbb{R}^{n}\right)$ with (24). Let $f \in B_{p q}^{s, m l o c}\left(\mathbb{R}^{n}, \boldsymbol{w}\right)$, then we get from the Lemma 2

$$
f=f * \theta_{0} * \psi_{0}+\sum_{\nu=1}^{\infty} 2^{\nu n} \theta\left(2^{\nu} \cdot\right) * \psi_{\nu} * f,
$$

where $\psi_{\nu}(\cdot)=2^{\nu n} \psi\left(2^{\nu} \cdot\right)$. Now, splitting the integration with respect to the cubes $Q_{\nu m}$ we derive

$$
\begin{aligned}
f(x)= & \sum_{m \in \mathbb{Z}^{n}} \int_{Q_{0 m}} \theta_{0}(x-y)\left(\psi_{0} * f\right)(y) d y \\
& +\sum_{\nu=1}^{\infty} \sum_{m \in \mathbb{Z}^{n}} 2^{\nu n} \int_{Q_{\nu m}} \theta\left(2^{\nu}(x-y)\right)\left(\psi_{\nu} * f\right)(y) d y .
\end{aligned}
$$

We define for each $\nu \in \mathbb{N}$ and all $m \in \mathbb{Z}^{n}$

$$
\lambda_{\nu m}=C_{\theta} \sup _{y \in Q_{\nu m}}\left|\left(\psi_{\nu} * f\right)(y)\right|,
$$

where $C_{\theta}=\max \left\{\sup _{|x| \leq 1}\left|D^{\beta} \theta(x)\right|:|\beta| \leq K\right\}$. If $\lambda_{\nu m} \neq 0$, then we define

$$
a_{\nu m}(x)=\frac{1}{\lambda_{\nu m}} 2^{\nu n} \int_{Q_{\nu m}} \theta\left(2^{\nu}(x-y)\right)\left(\psi_{\nu} * f\right)(y) d y,
$$

otherwise we set $a_{\nu m}(x)=0$. The $a_{0 m}$ atoms and $\lambda_{0 m}$ are defined similarly using $\theta_{0}$ and $\psi_{0}$. Clearly, (25) and the properties of $\theta_{0}, \psi_{0}, \theta$ and $\psi_{\nu}$ ensure that $a_{\nu m}$ are $[K, L]$-atoms. It remains to prove, that there exists a constant $c$ such that $\left\|\lambda\left|b_{p q}^{s, m l o c}(\boldsymbol{w})\|\leq c\| f\right| B_{p q}^{s, m l o c}\left(\mathbb{R}^{n}, \boldsymbol{w}\right)\right\|$. We have for fixed $\nu \in \mathbb{N}_{0}$ and $a>\frac{n}{p}+\alpha$

$$
\begin{aligned}
\sum_{m \in \mathbb{Z}^{n}} w_{\nu}(x) \lambda_{\nu m} \chi_{\nu m}(x) & \leq c \sum_{m \in \mathbb{Z}^{n}} w_{\nu}(x) \sup _{y \in Q_{\nu m}}\left|\left(\psi_{\nu} * f\right)(y)\right| \chi_{\nu m}(x) \\
& \leq c^{\prime} w_{\nu}(x) \sup _{|z| \leq c 2^{-\nu}} \frac{\left|\left(\psi_{\nu} * f\right)(x-z)\right|}{1+\left|2^{\nu} z\right|^{a}}\left(1+\left|2^{\nu} z\right|^{a}\right) \\
& \leq c^{\prime \prime} w_{\nu}(x)\left(\psi_{\nu}^{*} f\right)_{a}(x),
\end{aligned}
$$

since $|x-y| \leq c 2^{-\nu}$ for $x, y \in Q_{\nu m}$ and $\sum_{m \in \mathbb{Z}^{n}} \chi_{\nu m}(x)=1$. Here, $\left(\psi_{\nu}^{*} f\right)_{a}$ denotes the Peetre maximal operator, defined in (8). Therefore, we have 
using (12)

$$
\left\|\lambda \mid b_{p q}^{s, m l o c}(\boldsymbol{w})\right\| \leq c\left(\sum_{\nu=0}^{\infty} 2^{\nu s q}\left\|w_{\nu}(x)\left(\psi_{\nu}^{*} f\right)_{a}(x) \mid L_{p}\left(\mathbb{R}^{n}\right)\right\|^{q}\right)^{1 / q} .
$$

Since $\psi_{0} \in \mathcal{S}\left(\mathbb{R}^{n}\right)$ and $\psi \in \mathcal{S}\left(\mathbb{R}^{n}\right)$ are two kernels which fulfill the moment conditions (9) and the Tauberian conditions (10) and (11), we can use Proposition 3 with $a>\frac{n}{p}+\alpha$ and derive from (28)

$$
\left\|\lambda\left|b_{p q}^{s, m l o c}(\boldsymbol{w})\|\leq c\| f\right| B_{p q}^{s, m l o c}\left(\mathbb{R}^{n}, \boldsymbol{w}\right)\right\| .
$$

To prove the converse direction of the atomic decomposition, we take the more general molecules in the above sense. To this end, we need two technical lemmas as used in [5]. We want to estimate the size of $\varphi_{j}^{\vee} * \mu_{\nu m}$, where $\left\{\varphi_{j}\right\} \in \Phi\left(\mathbb{R}^{n}\right)$ and $\mu_{\nu m}$ is a $[K, L, M]$-molecule concentrated in $Q_{\nu m}$. We use the resolution of unity introduced in Remark 1. We recall Lemma 3.3 in [5].

Lemma 3. Let $\left\{\varphi_{j}\right\}_{j \in \mathbb{N}_{0}} \in \Phi\left(\mathbb{R}^{n}\right)$ be a resolution of unity and $\left\{\mu_{\nu m}\right\}_{\nu \in \mathbb{N}_{0}, m \in \mathbb{Z}^{n}}$ are $[K, L, M]$-molecules. Then we have for all $x \in \mathbb{R}^{n}$

$$
\left|\left(\varphi_{j}^{\vee} * \mu_{\nu m}\right)(x)\right| \leq c 2^{-(\nu-j)(L+n)}\left(1+2^{j}\left|x-2^{-\nu} m\right|\right)^{L+n-M} \quad, \quad \text { for } j \leq \nu
$$

and

$$
\left|\left(\varphi_{j}^{\vee} * \mu_{\nu m}\right)(x)\right| \leq c 2^{-(j-\nu) K}\left(1+2^{j}\left|x-2^{-\nu} m\right|\right)^{-M} \quad \text { for } j \geq \nu .
$$

The next lemma is analogous to Lemma 3.4 in [5] and the proof is in the Appendix.

Lemma 4. Let $1 \leq p \leq \infty, \boldsymbol{w} \in \mathcal{W}_{\alpha_{1}, \alpha_{2}}^{\alpha}$ and let

$$
F(x)=\sum_{m \in \mathbb{Z}^{n}} \lambda_{\nu m} f_{\nu m}
$$

where $\lambda_{\nu m} \in \mathbb{C}$ and $f_{\nu m}(x)=\left(1+2^{j}\left|x-2^{-\nu} m\right|\right)^{-R}$ with $R>\alpha+n$. Then we have for $j \leq \nu$

$$
\left\|F \mid L_{p}\left(\mathbb{R}^{n}, w_{j}\right)\right\| \leq c 2^{-\nu \frac{n}{p}} 2^{(\nu-j)\left(\alpha_{1}+n\right)}\left(\sum_{m \in \mathbb{Z}^{n}}\left|\lambda_{\nu m}\right|^{p} w_{\nu}^{p}\left(2^{-\nu} m\right)\right)^{1 / p}
$$


and for $j \geq \nu$

$$
\left\|F \mid L_{p}\left(\mathbb{R}^{n}, w_{j}\right)\right\| \leq c 2^{-\nu \frac{n}{p}} 2^{(j-\nu) \alpha_{2}}\left(\sum_{m \in \mathbb{Z}^{n}}\left|\lambda_{\nu m}\right|^{p} w_{\nu}^{p}\left(2^{-\nu} m\right)\right)^{1 / p} .
$$

Finally, we can state the converse direction of the decomposition theorem by molecules.

Theorem 2. Let $\boldsymbol{w} \in \mathcal{W}_{\alpha_{1}, \alpha_{2}}^{\alpha}, s \in \mathbb{R}$ and let $0<p, q \leq \infty$. Further, let $K, L \in \mathbb{N}_{0}$ with $K>s+\alpha_{2}, L>\sigma_{p}-s+\alpha_{1}$ and $M>0$ large enough $(M>L+n(\max (1,1 / p)+1)+2 \alpha)$. If $\left\{\mu_{\nu m}\right\}$ are $[K, L, M]$-molecules and $\lambda=\left\{\lambda_{\nu m}\right\} \in b_{p q}^{s, m l o c}(\boldsymbol{w})$, then

$$
f=\sum_{\nu=0}^{\infty} \sum_{m \in \mathbb{Z}^{n}} \lambda_{\nu m} \mu_{\nu m}, \quad \text { convergence being in } \mathcal{S}^{\prime}\left(\mathbb{R}^{n}\right),
$$

is an element of $B_{p q}^{s, m l o c}\left(\mathbb{R}^{n}, \boldsymbol{w}\right)$ and

$$
\left\|f\left|B_{p q}^{s, m l o c}\left(\mathbb{R}^{n}, \boldsymbol{w}\right)\|\leq c\| \lambda\right| b_{p q}^{s, m l o c}(\boldsymbol{w})\right\| .
$$

Proof. We have the representation of $f \in \mathcal{S}^{\prime}\left(\mathbb{R}^{n}\right)$ by (31) and we know by Lemma 1 that this representation converges. Now, we estimate the norm of $f$

$$
\begin{aligned}
\left\|f \mid B_{p q}^{s, m l o c}\left(\mathbb{R}^{n}, \boldsymbol{w}\right)\right\| & =\left(\sum_{j=0}^{\infty} 2^{j s q}\left\|\left(\varphi_{j} \hat{f}\right)^{\vee} w_{j} \mid L_{p}\left(\mathbb{R}^{n}\right)\right\|^{q}\right)^{1 / q} \\
\leq & c\left(\sum_{j=0}^{\infty} 2^{j s q}\left\|\sum_{\nu=0}^{\infty} \sum_{m \in \mathbb{Z}^{n}} \lambda_{\nu m}\left(\varphi_{j}^{\vee} * \mu_{\nu m}\right) w_{j} \mid L_{p}\left(\mathbb{R}^{n}\right)\right\|^{q}\right)^{1 / q} \\
\leq & c\left(\sum_{j=0}^{\infty} 2^{j s q}\left\|\sum_{\nu=0}^{j} \sum_{m \in \mathbb{Z}^{n}} \ldots \mid L_{p}\left(\mathbb{R}^{n}\right)\right\|^{q}\right)^{1 / q} \\
& +c\left(\sum_{j=0}^{\infty} 2^{j s q}\left\|\sum_{\nu=j+1}^{\infty} \sum_{m \in \mathbb{Z}^{n}} \ldots L_{p}\left(\mathbb{R}^{n}\right)\right\|^{q}\right)^{1 / q}=I_{i}+I I_{i} .
\end{aligned}
$$

Let $p \geq 1$. We estimate the first $L_{p}$ Norm in (32). We use (30) and we derive from Lemma 4 with $R=M>n+\alpha$ 


$$
\begin{aligned}
I_{j}: & =\left\|\sum_{\nu=0}^{j} \sum_{m \in \mathbb{Z}^{n}} \lambda_{\nu m}\left(\varphi_{j}^{\vee} * \mu_{\nu m}\right) w_{j} \mid L_{p}\left(\mathbb{R}^{n}\right)\right\| \\
& \leq c \sum_{\nu=0}^{j} 2^{-\nu \frac{n}{p}} 2^{-(j-\nu)\left(K-\alpha_{2}\right)}\left(\sum_{m \in \mathbb{Z}^{n}}\left|\lambda_{\nu m}\right|^{p} w_{\nu}^{p}\left(2^{-\nu} m\right)\right)^{1 / p} .
\end{aligned}
$$

For the second term in (32) we use (29) and Lemma 4 with $R=M-L-n>$ $n+\alpha$ and get

$$
\begin{aligned}
I I_{j}: & =\left\|\sum_{\nu=j+1}^{\infty} \sum_{m \in \mathbb{Z}^{n}} \lambda_{\nu m}\left(\varphi_{j}^{\vee} * \mu_{\nu m}\right) w_{j} \mid L_{p}\left(\mathbb{R}^{n}\right)\right\| \\
& \leq c \sum_{\nu=j+1}^{\infty} 2^{-\nu \frac{n}{p}} 2^{-(\nu-j)\left(L-\alpha_{1}\right)}\left(\sum_{m \in \mathbb{Z}^{n}}\left|\lambda_{\nu m}\right|^{p} w_{\nu}^{p}\left(2^{-\nu} m\right)\right)^{1 / p} .
\end{aligned}
$$

Now, we consider the case $0<p<1$. We use the embedding $\ell_{p} \hookrightarrow \ell_{1}$ and obtain for the first term in (32)

$$
\begin{aligned}
I_{j} & \leq\left(\int_{\mathbb{R}^{n}}\left(\sum_{\nu=0}^{j} \sum_{m \in \mathbb{Z}^{n}}\left|\lambda_{\nu m}\right|\left|\left(\varphi_{j}^{\vee} * \mu_{\nu m}\right)(x)\right| w_{j}(x)\right)^{p} d x\right)^{1 / p} \\
& \leq c\left(\sum_{\nu=0}^{j} \sum_{m \in \mathbb{Z}^{n}}\left|\lambda_{\nu m}\right|^{p} \int_{\mathbb{R}^{n}}\left|\left(\varphi_{j}^{\vee} * \mu_{\nu m}\right)(x)\right|^{p} w_{j}^{p}(x) d x\right)^{1 / p} .
\end{aligned}
$$

By applying (30) and using the properties of the weight sequence, we estimate

$$
\begin{aligned}
\int_{\mathbb{R}^{n}} & \left|\left(\varphi_{j}^{\vee} * \mu_{\nu m}\right)(x)\right|^{p} w_{j}^{p}(x) d x \\
& \leq c 2^{-(j-\nu)\left(K-\alpha_{2}\right) p} \int_{\mathbb{R}^{n}}\left(1+2^{\nu}\left|x-2^{-\nu} m\right|\right)^{(-M+\alpha) p} w_{\nu}^{p}\left(2^{-\nu} m\right) d x \\
& \leq c^{\prime} 2^{-(j-\nu)\left(K-\alpha_{2}\right) p} 2^{-\nu n} w_{\nu}^{p}\left(2^{-\nu} m\right), \quad \text { for } M>\frac{n}{p}+\alpha .
\end{aligned}
$$

So, we get for the first $L_{p}$ norm

$$
I_{j} \leq c\left(\sum_{\nu=0}^{j} 2^{-\nu n} 2^{-(j-\nu)\left(K-\alpha_{2}\right) p} \sum_{m \in \mathbb{Z}^{n}}\left|\lambda_{\nu m}\right|^{p} w_{\nu}^{p}\left(2^{-\nu} m\right)\right)^{1 / p} .
$$


By a similar calculation we obtain the second estimate $\left(M>\frac{n}{p}+L+n+\alpha\right)$

$$
I I_{j} \leq c\left(\sum_{\nu=j+1}^{\infty} 2^{-\nu n} 2^{-(\nu-j)\left(L-\sigma_{p}-\alpha_{1}\right) p} \sum_{m \in \mathbb{Z}^{n}}\left|\lambda_{\nu m}\right|^{p} w_{\nu}^{p}\left(2^{-\nu} m\right)\right)^{1 / p} .
$$

We denote $\tilde{p}:=\min (1, p)$ and $t:=\min (1, p, q)$. We can rewrite our results for the first term as

$$
I_{j} \leq c\left(\sum_{\nu=0}^{j} 2^{-\nu \frac{n}{p} \tilde{p}} 2^{-(j-\nu)\left(K-\alpha_{2}\right) \tilde{p}}\left(\sum_{m \in \mathbb{Z}^{n}}\left|\lambda_{\nu m}\right|^{p} w_{\nu}^{p}\left(2^{-\nu} m\right)\right)^{\tilde{p} / p}\right)^{1 / \tilde{p}}
$$

for all $0<p, q \leq \infty$. Finally, we conclude with that notation and $\ell_{t / \tilde{p}} \hookrightarrow \ell_{1}$

$$
\left\|2^{j s} I_{j}\left|\ell_{q}\left\|^{t} \leq c\right\|\right| \sum_{\nu=0}^{j} 2^{\nu\left(s-\frac{n}{p}\right) t} 2^{-(j-\nu)\left(K-s-\alpha_{2}\right) t}\left(\sum_{m \in \mathbb{Z}^{n}}\left|\lambda_{\nu m}\right|^{p} w_{\nu}^{p}\left(2^{-\nu} m\right)\right)^{t / p} \mid \ell_{q / t}\right\|,
$$

and Young's inequality gives us

$$
\begin{aligned}
& \leq c^{\prime}\left(\sum_{\nu=0}^{\infty} 2^{\nu\left(s-\frac{n}{p}\right) q}\left(\sum_{m \in \mathbb{Z}^{n}}\left|\lambda_{\nu m}\right|^{p} w_{\nu}^{p}\left(2^{-\nu} m\right)\right)^{q / p}\right)^{t / q}\left(\sum_{j=0}^{\infty} 2^{-j \zeta t}\right) \\
& =c^{\prime \prime}\left\|\lambda \mid b_{p q}^{s, m l o c}(\boldsymbol{w})\right\|^{t}, \quad \text { where } \zeta:=K-s-\alpha_{2}>0 .
\end{aligned}
$$

With the same notation a similar estimate can be achieved for $\left\|2^{j s} I I_{j} \mid \ell_{q}\right\|^{t}$. Here one has to use $\zeta:=L-\sigma_{p}+s-\alpha_{1}>0$ and this finishes the proof.

For every $M>0$ every $[K, L]$ atom is a $[K, L, M]$ molecule. So we get an easy corollary for the atomic decomposition.

Corollary 1. Let $\boldsymbol{w}=\left\{w_{k}\right\}_{k \in \mathbb{N}_{0}} \in \mathcal{W}_{\alpha_{1}, \alpha_{2}}^{\alpha}$ and let $0<p, q \leq \infty, s \in \mathbb{R}$.

Furthermore, let $K, L \in \mathbb{N}_{0}$ with $K>s+\alpha_{2}$ and $L>\sigma_{p}-s+\alpha_{1}$.

(i) If $\lambda \in b_{p q}^{s, m l o c}(\boldsymbol{w})$ and $\left\{a_{\nu m}\right\}_{\nu \in \mathbb{N}_{0}, m \in \mathbb{Z}^{n}}$ are $[K, L]$-atoms centered at $Q_{\nu m}$, then

$$
f=\sum_{\nu=0}^{\infty} \sum_{m \in \mathbb{Z}^{n}} \lambda_{\nu m} a_{\nu m}
$$


144 Decompositions of generalized 2-microlocal Besov spaces

belongs to the space $B_{p q}^{s, m l o c}\left(\mathbb{R}^{n}, \boldsymbol{w}\right)$ and there exists a constant $c>0$ with

$$
\left\|f\left|B_{p q}^{s, m l o c}\left(\mathbb{R}^{n}, \boldsymbol{w}\right)\|\leq c\| \lambda\right| b_{p q}^{s, m l o c}(\boldsymbol{w})\right\|
$$

The constant $c$ is universal for all $\lambda$ and $a_{\nu m}$.

(ii) For each $f \in B_{p q}^{s, m l o c}\left(\mathbb{R}^{n}, \boldsymbol{w}\right)$ there exist $\lambda \in b_{p q}^{s, m l o c}(\boldsymbol{w})$ and $[K, L]$ atoms centered at $Q_{\nu m}$ such that $f$ can be represented as in (33) (convergence being in $\mathcal{S}^{\prime}\left(\mathbb{R}^{n}\right)$ ), where

$$
\left\|\lambda\left|b_{p q}^{s, m l o c}(\boldsymbol{w})\|\leq c\| f\right| B_{p q}^{s, m l o c}\left(\mathbb{R}^{n}, \boldsymbol{w}\right)\right\|
$$

and the constant $c$ is universal for all $f \in B_{p q}^{s, m l o c}\left(\mathbb{R}^{n}, \boldsymbol{w}\right)$.

\section{Wavelet decomposition}

In this section we describe the characterization of $B_{p q}^{s, m l o c}\left(\mathbb{R}^{n}, \boldsymbol{w}\right)$ by a decomposition in wavelets. We follow closely the ideas expressed in [19], [20] and [11].

5.1 Preliminaries. First of all, we recall some results from wavelet theory.

Proposition 5. (i) There are a real scaling function $\psi_{F} \in \mathcal{S}(\mathbb{R})$ and a real associated wavelet $\psi_{M} \in \mathcal{S}(\mathbb{R})$ such that their Fourier transforms have compact supports, $\widehat{\psi_{F}}(0)=(2 \pi)^{-1 / 2}$ and

$$
\operatorname{supp} \widehat{\psi_{M}} \subseteq\left[-\frac{8}{3} \pi,-\frac{2}{3} \pi\right] \cup\left[\frac{2}{3} \pi, \frac{8}{3} \pi\right] .
$$

(ii) For any $k \in \mathbb{N}$ there are a real compactly supported scaling function $\psi_{F} \in C^{k}(\mathbb{R})$ and a real compactly supported associated wavelet $\psi_{M} \in C^{k}(\mathbb{R})$ such that $\widehat{\psi_{F}}(0)=(2 \pi)^{-1 / 2}$ and

$$
\int_{\mathbb{R}} x^{l} \psi_{M}(x) d x=0 \quad \text { for all } l \in\{0, \ldots, k-1\} .
$$

In both cases we have, that $\left\{\psi_{\nu m}: \nu \in \mathbb{N}_{0}, m \in \mathbb{Z}\right\}$ is an orthonormal basis in $L_{2}(\mathbb{R})$, where

$$
\psi_{\nu m}(t):= \begin{cases}\psi_{F}(t-m), & \text { if } \nu=0, m \in \mathbb{Z} \\ 2^{\frac{\nu-1}{2}} \psi_{M}\left(2^{\nu-1} t-m\right), & \text { if } \nu \in \mathbb{N}, m \in \mathbb{Z}\end{cases}
$$

and the functions $\psi_{M}, \psi_{F}$ are according to $(i)$ or $(i i)$. 
This Proposition is taken over from [21, Theorem 1.61]. The wavelets in the first part are called Meyer wavelets. They do not have a compact support but they are rapidly decaying functions $\left(\psi_{F}, \psi_{M} \in \mathcal{S}(\mathbb{R})\right)$ and $\psi_{M}$ has infinitely many moment conditions. The wavelets from the second part are called Daubechies wavelets. Here the functions $\psi_{M}, \psi_{F}$ do have compact support, but they have only limited smoothness. Both types of wavelets are well described in [22], chapters 3 and 4.

This orthonormal basis can be generalized to the $n$-dimensional case by a tensor product procedure. We take over the notation from [21, Subsection 4.2.1] with $l=0$. Let $\psi_{M}, \psi_{F}$ be the Meyer or Daubechies wavelets described above. Now, we define

$$
G^{0}=\{F, M\}^{n} \quad \text { and } \quad G^{\nu}=\{F, M\}^{n *} \text { if } \nu \geq 1,
$$

where the * indicates, that at least one $G_{i}$ of $G=\left(G_{1}, \ldots, G_{n}\right) \in\{F, M\}^{n *}$ must be an $M$. It is clear from the definition, that the cardinal number of $\{F, M\}^{n *}$ is $2^{n}-1$. Let for $x \in \mathbb{R}$

$$
\Psi_{G m}^{\nu}(x)=2^{v \frac{n}{2}} \prod_{r=1}^{n} \psi_{G_{r}}\left(2^{\nu} x_{r}-m_{r}\right),
$$

where $G \in G^{\nu}, m \in \mathbb{Z}^{n}$ and $\nu \in \mathbb{N}_{0}$. Then $\left\{\Psi_{G m}^{\nu}: \nu \in \mathbb{N}_{0}, G \in G^{\nu}, m \in\right.$ $\left.\mathbb{Z}^{n}\right\}$ is an orthonormal basis in $L_{2}\left(\mathbb{R}^{n}\right)$. Finally, we have to adjust the sequence spaces $b_{p q}^{s, m l o c}(\boldsymbol{w})$ to our situation.

Definition 7. Let $\boldsymbol{w} \in \mathcal{W}_{\alpha_{1}, \alpha_{2}}^{\alpha}, s \in \mathbb{R}$ and $0<p, q \leq \infty$. Then

$$
\tilde{b}_{p q}^{s, m l o c}(\boldsymbol{w}):=\left\{\lambda=\left\{\lambda_{G m}^{\nu}\right\} \subset \mathbb{C}:\left\|\lambda \mid \tilde{b}_{p q}^{s, m l o c}(\boldsymbol{w})\right\|<\infty\right\},
$$

where

$$
\left\|\lambda \mid \tilde{b}_{p q}^{s, m l o c}(\boldsymbol{w})\right\|=\left(\sum_{\nu=0}^{\infty} 2^{\nu\left(s-\frac{n}{p}\right) q} \sum_{G \in G^{\nu}}\left(\sum_{m \in \mathbb{Z}^{n}}\left|\lambda_{G m}^{\nu}\right|^{p} w_{\nu}^{p}\left(2^{-\nu} m\right)\right)^{q / p}\right)^{1 / q} .
$$

To get the wavelet characterization we use local means with kernels which only have limited smoothness and we use the molecular decomposition described in the previous section. This idea goes back to [20], [11] and [7]. First, we recall the local means with kernel $k$

$$
k(t, f)(x)=t^{-n} \int_{\mathbb{R}^{n}} k\left(\frac{y-x}{t}\right) f(y) d y .
$$


With $t=2^{-j}, x=2^{-j} l$ where $j \in \mathbb{N}_{0}$ and $l \in \mathbb{Z}^{n}$, one gets

$$
\begin{aligned}
k\left(2^{-j}, f\right)\left(2^{-j} l\right) & =2^{j n} \int_{\mathbb{R}^{n}} k\left(2^{j} y-l\right) f(y) d y \\
& =\int_{\mathbb{R}^{n}} k_{j l}(y) f(y) d y \\
& =k_{j l}(f) .
\end{aligned}
$$

First, assume that the expression (35) makes sense, at least formally. Later on we show that (35) is a dual pairing. Now, the usual properties for $k$ are transferred to the kernels $k_{j l}$.

Definition 8. Let $k_{j l}(x) \in C^{A}\left(\mathbb{R}^{n}\right)$ with $j \in \mathbb{N}_{0}$ and $l \in \mathbb{Z}^{n}$ be functions in $R^{n}$ with

$$
\left|D^{\beta} k_{j l}(x)\right| \leq c 2^{j|\beta|+j n}\left(1+2^{j}\left|x-2^{-j} l\right|\right)^{-C}, \quad|\beta| \leq A \in \mathbb{N}_{0}, C>0,
$$

for all $x \in \mathbb{R}^{n}, j \in \mathbb{N}_{0}, l \in \mathbb{Z}^{n}$ and $\int_{\mathbb{R}^{n}} x^{\beta} k_{j l}(x) d x=0, \quad|\beta|<B \in \mathbb{N}_{0}$, for $j \geq 1$ and $l \in \mathbb{Z}^{n}$.

It is clear from the definition, that $\left\{2^{-j n} k_{j l}\right\}$ are $[A, B, C]$ molecules.

5.2 Duality. In this subsection we show that the expression

$$
k(f)=\int_{\mathbb{R}^{n}} k(y) f(y) d y
$$

makes sense as a dual pairing. Here, $\boldsymbol{w} \in \mathcal{W}_{\alpha_{1}, \alpha_{2}}^{\alpha}, f \in B_{p q}^{s, m l o c}\left(\mathbb{R}^{n}, \boldsymbol{w}\right)$ and the function $k: \mathbb{R}^{n} \mapsto \mathbb{C}$ belongs to some weighted space of continuously differentiable functions $C^{u}\left(\mathbb{R}^{n}, \kappa\right), \kappa \geq 0$,

$C^{u}\left(\mathbb{R}^{n}, \kappa\right):=\left\{f \in C^{u}\left(\mathbb{R}^{n}\right):(1+|x|)^{\kappa} D^{\beta} f(x) \in C\left(\mathbb{R}^{n}\right)\right.$ for all $\left.|\beta| \leq u\right\}$,

normed by

$$
\left\|f\left|C^{u}\left(\mathbb{R}^{n}, \kappa\right) \|=\max _{|\beta| \leq u} \sup _{x \in \mathbb{R}^{n}}(1+|x|)^{\kappa}\right| D^{\beta} f(x) \mid .\right.
$$

We need to introduce the dual space of $B_{p q}^{s, m l o c}\left(\mathbb{R}^{n}, \boldsymbol{w}\right)$. For $s \in \mathbb{R}$ and $\max (p, q)<\infty$, we have that $\mathcal{S}\left(\mathbb{R}^{n}\right)$ is dense in $B_{p q}^{s, m l o c}\left(\mathbb{R}^{n}, \boldsymbol{w}\right)$. Therefore, a linear functional on $B_{p q}^{s, m l o c}\left(\mathbb{R}^{n}, \boldsymbol{w}\right)$ can be interpreted as an element of $\mathcal{S}^{\prime}\left(\mathbb{R}^{n}\right)$. That means, $g \in \mathcal{S}^{\prime}\left(\mathbb{R}^{n}\right)$ belongs to the dual space $\left(B_{p q}^{s, m l o c}\left(\mathbb{R}^{n}, \boldsymbol{w}\right)\right)^{\prime}$ of the space $B_{p q}^{s, m l o c}\left(\mathbb{R}^{n}, \boldsymbol{w}\right)$, if and only if there exists a 
positive number $c$ such that

$$
|g(\varphi)| \leq c\left\|\varphi \mid B_{p q}^{s, m l o c}\left(\mathbb{R}^{n}, \boldsymbol{w}\right)\right\| \quad \text { for all } \varphi \in \mathcal{S}\left(\mathbb{R}^{n}\right) .
$$

We do not want to give a full treatment of duality theory in Besov spaces, but one can modify the statements and proofs in Section 2.11 in [18] and one derives at least

$$
B_{p^{\prime} q^{\prime}}^{\sigma_{p}-s, m l o c}\left(\mathbb{R}^{n}, \boldsymbol{w}^{-\mathbf{1}}\right) \hookrightarrow\left(B_{p q}^{s, m l o c}\left(\mathbb{R}^{n}, \boldsymbol{w}\right)\right)^{\prime},
$$

where $\sigma_{p}=n(1 / p-1)_{+}$and

$$
\frac{1}{p}+\frac{1}{p^{\prime}}=1 \quad \text { if } 1 \leq p<\infty \quad \text { and } \quad p^{\prime}=\infty \text { if } 0<p<1 .
$$

Similarly for $q^{\prime}$. It is easy to see, that $\boldsymbol{w}^{-\mathbf{1}}=\left\{\frac{1}{w_{j}}\right\}_{j \in \mathbb{N}_{0}} \in \mathcal{W}_{\alpha_{2}, \alpha_{1}}^{\alpha}$ for $\boldsymbol{w} \in \mathcal{W}_{\alpha_{1}, \alpha_{2}}^{\alpha}$.

For $\max (p, q)=\infty$ we get a similar result, where the right hand side of (37) is replaced by $\left(\stackrel{\circ}{B}_{p q}^{s, m l o c}\left(\mathbb{R}^{n}, \boldsymbol{w}\right)\right)^{\prime}$, where $\stackrel{\circ}{B}_{p q}^{s, m l o c}\left(\mathbb{R}^{n}, \boldsymbol{w}\right)$ is the completion of $\mathcal{S}\left(\mathbb{R}^{n}\right)$ in $B_{p q}^{s, m l o c}\left(\mathbb{R}^{n}, \boldsymbol{w}\right)$. In this case we only have the dual pairing (35) for the Meyer wavelets, because they are elements of $\mathcal{S}\left(\mathbb{R}^{n}\right)$ and for the Daubechies wavelets, because they have a compact support. In the case $\max (p, q)<\infty$ we can extend this to more general wavelet bases. The wavelets belong to $C^{u}\left(\mathbb{R}^{n}, \kappa\right)$ and we show under which conditions on $u \in \mathbb{N}_{0}$ and $\kappa \geq 0$ we have

$$
C^{u}\left(\mathbb{R}^{n}, \kappa\right) \hookrightarrow B_{p^{\prime} q^{\prime}}^{\sigma_{p}-s, m l o c}\left(\mathbb{R}^{n}, \boldsymbol{w}^{-\mathbf{1}}\right) .
$$

First of all, we show $C^{0}\left(\mathbb{R}^{n}, \kappa\right) \hookrightarrow B_{p^{\prime} \infty}^{-\alpha_{1}, m l o c}\left(\mathbb{R}^{n}, \boldsymbol{w}^{-\mathbf{1}}\right)$. We use our fixed resolution of unity from Remark 1, the weight properties and Young's inequality to see that

$$
\begin{aligned}
\left\|f \mid B_{p^{\prime} \infty}^{-\alpha_{1}, m l o c}\left(\mathbb{R}^{n}, \boldsymbol{w}^{-1}\right)\right\| & =\sup _{j \in \mathbb{N}_{0}} 2^{-\alpha_{1} j}\left\|w_{j}^{-1}\left(\varphi_{j} \hat{f}\right)^{\vee} \mid L_{p^{\prime}}\left(\mathbb{R}^{n}\right)\right\| \\
& \leq c \sup _{j \in \mathbb{N}_{0}}\left\|w_{0}^{-1}\left(\varphi_{j}^{\vee} * f\right) \mid L_{p^{\prime}}\left(\mathbb{R}^{n}\right)\right\| \\
& \leq c^{\prime} \sup _{j \in \mathbb{N}_{0}}\left\|\left(w_{0}^{-1} \varphi_{j}^{\vee}\right) *\left((1+|\cdot|)^{\alpha} f\right) \mid L_{p^{\prime}}\left(\mathbb{R}^{n}\right)\right\| \\
& \leq c^{\prime \prime}\left\|(1+|\cdot|)^{\alpha} f\left|L_{p^{\prime}}\left(\mathbb{R}^{n}\right)\left\|\sup _{j \in \mathbb{N}_{0}}\right\| \varphi_{j}^{\vee}\right| L_{1}\left(\mathbb{R}^{n}\right)\right\| \\
& \leq c^{\prime \prime \prime}\left\|f \mid C^{0}\left(\mathbb{R}^{n}, \kappa\right)\right\|,
\end{aligned}
$$


where the last inequality comes from $\varphi_{j}=\varphi\left(2^{-j} \cdot\right) \in \mathcal{S}\left(\mathbb{R}^{n}\right)$ and that $\kappa>\frac{n}{p^{\prime}}+\alpha$. Let us assume now, that $f \in C^{u}\left(\mathbb{R}^{n}, \kappa\right)$, then by applying Proposition 1 we conclude

$$
C^{u}\left(\mathbb{R}^{n}, \kappa\right) \hookrightarrow B_{p^{\prime} \infty}^{u-\alpha_{1}, m l o c}\left(\mathbb{R}^{n}, \boldsymbol{w}^{-\mathbf{1}}\right)
$$

Finally, Proposition 4 gives us for $u>\sigma_{p}-s+\alpha_{1}$

$$
C^{u}\left(\mathbb{R}^{n}, \kappa\right) \hookrightarrow B_{p^{\prime} q^{\prime}}^{\sigma_{p}-s, m l o c}\left(\mathbb{R}^{n}, \boldsymbol{w}^{-\mathbf{1}}\right)
$$

Because of (37) and (39) the equation (36) makes sense at least for $u>\sigma_{p}-s+\alpha_{1}$.

Further, we mention that all functions $\left\{k_{j l}\right\}$ from Definition 8 with $A \geq u, B \in \mathbb{N}_{0}$ arbitrary and $C \geq \kappa$ belong to the space $C^{u}\left(\mathbb{R}^{n}, \kappa\right)$. So we see that (35) is well defined for $A>\sigma_{p}-s+\alpha_{1}$ and $C \geq \alpha+n$, but these conditions will always be fulfilled in the following theorems.

5.3 Wavelet isomorphism. We want to use the molecular decomposition obtained in the last section. We assume, that $\left\{\mu_{\nu m}\right\}$ are $[K, L, M]$ molecules and that the $\left\{k_{j l}\right\}$ are the above given functions from Definition 8. Before stating the theorem we have to prove some fundamental lemmas. First, we have to give estimates of the quantity $\left|\left\langle\mu_{\nu m}, k_{j l}\right\rangle\right|$. The proofs go back to [6, Appendix B].

Lemma 5. (i) Let $\nu \geq j, M>A+n$ and $L \geq A$, then

$$
\left|\left\langle\mu_{\nu m}, k_{j l}\right\rangle\right| \leq c 2^{-(\nu-j)(A+n)}\left(1+2^{j}\left|2^{-\nu} m-2^{-j} l\right|\right)^{-\min (M-A-n, C)} .
$$

(ii) Let $\nu \leq j, C>K+n$ and $B \geq K$, then

$$
\left|\left\langle\mu_{\nu m}, k_{j l}\right\rangle\right| \leq c 2^{-(j-\nu) K}\left(1+2^{\nu}\left|2^{-\nu} m-2^{-j} l\right|\right)^{-\min (M, C-K-n)} .
$$

The next lemma is presented in [11, Lemma 7.4]. Nevertheless, we present a proof in the Appendix because our notation is a bit different.

Lemma 6. Let $1 \leq p \leq \infty$ and $b_{m} \in \mathbb{C}$.

(i) Let $\nu \geq j, R>n$ and $\frac{1}{p}+\frac{1}{p^{\prime}}=1$. Then

$$
\left(\sum_{l \in \mathbb{Z}^{n}}\left(\sum_{m \in \mathbb{Z}^{n}}\left|b_{m}\right|\left(1+2^{j}\left|2^{-j} l-2^{-\nu} m\right|\right)^{-R}\right)^{p}\right)^{1 / p} \leq c 2^{(\nu-j) \frac{n}{p^{\prime}}}\left(\sum_{m \in \mathbb{Z}^{n}}\left|b_{m}\right|^{p}\right)^{1 / p}
$$


(ii) Let $\nu \leq j$ and $R>n$. Then

$$
\left(\sum_{l \in \mathbb{Z}^{n}}\left(\sum_{m \in \mathbb{Z}^{n}}\left|b_{m}\right|\left(1+2^{\nu}\left|2^{-j} l-2^{-\nu} m\right|\right)^{-R}\right)^{p}\right)^{1 / p} \leq c 2^{(j-\nu) \frac{n}{p}}\left(\sum_{m \in \mathbb{Z}^{n}}\left|b_{m}\right|^{p}\right)^{1 / p} .
$$

Now, we are ready to state the first theorem, which gives us one direction of the wavelet decomposition. We define

$$
k(f)=\left\{k_{j l}(f): j \in \mathbb{N}_{0}, l \in \mathbb{Z}^{n}\right\}
$$

Theorem 3. Let $w \in \mathcal{W}_{\alpha_{1}, \alpha_{2}}^{\alpha}, s \in \mathbb{R}$ and $0<p, q \leq \infty$. Further, let $\left\{k_{j l}\right\}$ be as in Definition 8 with $C>0$ large enough and $A, B \in \mathbb{N}_{0}$ with

$$
A>\sigma_{p}-s+\alpha_{1}, \quad B>s+\alpha_{2} .
$$

Then for some $c>0$ and all $f \in B_{p q}^{s, m l o c}\left(\mathbb{R}^{n}, \boldsymbol{w}\right)$,

$$
\left\|k(f)\left|b_{p q}^{s, m l o c}(\boldsymbol{w})\|\leq c\| f\right| B_{p q}^{s, m l o c}\left(\mathbb{R}^{n}, \boldsymbol{w}\right)\right\| .
$$

Proof. We assume $f \in B_{p q}^{s, m l o c}\left(\mathbb{R}^{n}, \boldsymbol{w}\right)$ and we use the atomic decomposition theorem (Corollary 1 ) to get

$$
f=\sum_{\nu=0}^{\infty} \sum_{m \in \mathbb{Z}^{n}} \lambda_{\nu m} a_{\nu m}
$$

where $\left\{a_{\nu m}\right\}$ are $[K, L]$ atoms with $K=B>s+\alpha_{2}$ and $L=A>$ $\sigma_{p}-s+\alpha_{1}$. We want to show

$$
\left\|k(f)\left|b_{p q}^{s, m l o c}(\boldsymbol{w})\|\leq c\| \lambda\right| b_{p q}^{s, m l o c}(\boldsymbol{w})\right\| \leq c^{\prime}\left\|f \mid B_{p q}^{s, m l o c}\left(\mathbb{R}^{n}, \boldsymbol{w}\right)\right\|,
$$

where the last inequality comes from the atomic decomposition theorem. We recall, that atoms have compact support and that they are $[K, L, M]$ molecules for every $M>0$. We split (42) dependent on $j \in \mathbb{N}_{0}$ into

$$
\begin{aligned}
f & =f_{j}+f^{j}=\sum_{\nu=0}^{j} \cdots+\sum_{\nu=j+1}^{\infty} \cdots \quad \text { and get } \\
k_{j l}(f) & =\int_{\mathbb{R}^{n}} k_{j l}(y) f(y) d y=\int_{\mathbb{R}^{n}} k_{j l}(y) f_{j}(y) d y+\int_{\mathbb{R}^{n}} k_{j l}(y) f^{j}(y) d y .
\end{aligned}
$$

To shorten notation, we define $A(m, l):=\left(1+\min \left(2^{\nu}, 2^{j}\right)\left|2^{-\nu} m-2^{-j} l\right|\right)$. Let $\nu \leq j$ and $1 \leq p \leq \infty$, then we get by Minkowski's inequality and 
Lemma 5

$$
\begin{aligned}
& \left(\sum_{l \in \mathbb{Z}^{n}}\left|\int_{\mathbb{R}^{n}} k_{j l}(y) f_{j}(y) d y\right|^{p} w_{j}^{p}\left(2^{-j} l\right)\right)^{1 / p} \\
& \quad \leq \sum_{\nu=0}^{j}\left(\sum_{l \in \mathbb{Z}^{n}}\left(\sum_{m \in \mathbb{Z}^{n}}\left|\lambda_{\nu m}\right| w_{j}\left(2^{-j} l\right)\left|\left\langle k_{j l}, a_{\nu m}\right\rangle\right|\right)^{p}\right)^{1 / p} \\
& (44) \quad \leq c \sum_{\nu=0}^{j} 2^{-(j-\nu)\left(B-\alpha_{2}\right)}\left(\sum_{l \in \mathbb{Z}^{n}}\left(\sum_{m \in \mathbb{Z}^{n}}\left|\lambda_{\nu m}\right| w_{\nu}\left(2^{-\nu} m\right) A(m, l)^{-C+\alpha}\right)^{p}\right)^{1 / p} \\
& (45) \quad \leq c^{\prime} \sum_{\nu=0}^{j} 2^{-(j-\nu)\left(K-\alpha_{2}-\frac{n}{p}\right)}\left(\sum_{m \in \mathbb{Z}^{n}}\left|\lambda_{\nu m}\right|^{p} w_{\nu}^{p}\left(2^{-\nu} m\right)\right)^{1 / p},
\end{aligned}
$$

where in the last step we used Lemma 6 with $R=C-\alpha>n$. In the case $0<p<1$ we have also the estimate (44) and get by $\ell_{p} \hookrightarrow \ell_{1}$

$$
\left(\sum_{l \in \mathbb{Z}^{n}}\left|\int_{\mathbb{R}^{n}} k_{j l}(y) f_{j}(y) d y\right|^{p} w_{j}^{p}\left(2^{-j} l\right)\right)^{1 / p}
$$

$$
\leq c \sum_{\nu=0}^{j} 2^{-(j-\nu)\left(B-\alpha_{2}\right)}\left(\sum_{m \in \mathbb{Z}^{n}}\left|\lambda_{\nu m}\right|^{p} w_{\nu}^{p}\left(2^{-\nu} m\right) \sum_{l \in \mathbb{Z}^{n}} A(m, l)^{-p(C-\alpha)}\right)^{1 / p} .
$$

A direct calculation shows, that $\sum_{l \in \mathbb{Z}^{n}} A(m, l)^{-p(C-\alpha)} \leq c 2^{(j-\nu) n}$ for $C>\frac{n}{p}+\alpha$. Therefore, we get from (45) and (46)

$$
2^{j\left(s-\frac{n}{p}\right)}\left(\sum_{l \in \mathbb{Z}^{n}}\left|\int_{\mathbb{R}^{n}} k_{j l}(y) f_{j}(y) d y\right|^{p} w_{j}^{p}\left(2^{-j} l\right)\right)^{1 / p}
$$

$$
\leq c \sum_{\nu=0}^{j} 2^{\nu\left(s-\frac{n}{p}\right)} 2^{-(j-\nu) \zeta}\left(\sum_{m \in \mathbb{Z}^{n}}\left|\lambda_{\nu m}\right|^{p} w_{\nu}^{p}\left(2^{-\nu} m\right)\right)^{1 / p}
$$

where $\zeta=B-s-\alpha_{2}>0$. 
Let us consider the case $\nu>j$ and $1 \leq p \leq \infty$. Then we derive similar to the first case with Minkowski's inequality and Lemma 5

$$
\begin{aligned}
& \left(\sum_{l \in \mathbb{Z}^{n}}\left|\int_{\mathbb{R}^{n}} k_{j l}(y) f^{j}(y) d y\right|^{p} w_{j}^{p}\left(2^{-j} l\right)\right)^{1 / p} \\
& \quad \leq \sum_{\nu=j+1}^{\infty}\left(\sum_{l \in \mathbb{Z}^{n}}\left(\sum_{m \in \mathbb{Z}^{n}}\left|\lambda_{\nu m}\right| w_{j}\left(2^{-j} l\right)\left|\left\langle k_{j l}, a_{\nu m}\right\rangle\right|\right)^{p}\right)^{1 / p} \\
& (48) \\
& \quad \leq c \sum_{\nu=j+1}^{\infty} 2^{-(\nu-j)\left(A+n-\alpha_{1}\right)}\left(\sum_{l \in \mathbb{Z}^{n}}\left(\sum_{m \in \mathbb{Z}^{n}}\left|\lambda_{\nu m}\right| w_{\nu}\left(2^{-\nu} m\right) A(m, l)^{-C+A+n+\alpha}\right)^{p}\right)^{1 / p} \\
& \quad \leq c^{\prime} \sum_{\nu=j+1}^{\infty} 2^{-(\nu-j)\left(A-\alpha_{1}+\frac{n}{p}\right)}\left(\sum_{m \in \mathbb{Z}^{n}}\left|\lambda_{\nu m}\right|^{p} w_{\nu}^{p}\left(2^{-\nu} m\right)\right)^{1 / p},
\end{aligned}
$$

where we used Lemma 6 with $R=C-A-n-\alpha>n$ in the last step. We obtain now with $\rho=A+s-\alpha_{1}>0$

$$
\begin{aligned}
& 2^{j\left(s-\frac{n}{p}\right)}\left(\sum_{l \in \mathbb{Z}^{n}}\left|\int_{\mathbb{R}^{n}} k_{j l}(y) f^{j}(y) d y\right|^{p} w_{j}^{p}\left(2^{-j} l\right)\right)^{1 / p} \\
& \leq c \sum_{\nu=j+1}^{\infty} 2^{\nu\left(s-\frac{n}{p}\right)} 2^{-(\nu-j) \rho}\left(\sum_{m \in \mathbb{Z}^{n}}\left|\lambda_{\nu m}\right|^{p} w_{\nu}^{p}\left(2^{-\nu} m\right)\right)^{1 / p} .
\end{aligned}
$$

For $0<p<1$ we have again (48), use $\ell_{p} \hookrightarrow \ell_{1}$ and obtain

$$
\begin{aligned}
& \left(\sum_{l \in \mathbb{Z}^{n}}\left|\int_{\mathbb{R}^{n}} k_{j l}(y) f^{j}(y) d y\right|^{p} w_{j}^{p}\left(2^{-j} l\right)\right)^{1 / p} \\
& \quad \leq c \sum_{\nu=j+1}^{\infty} 2^{-(\nu-j)\left(A+n-\alpha_{1}\right)}\left(\sum_{m \in \mathbb{Z}^{n}}\left|\lambda_{\nu m}\right|^{p} w_{\nu}^{p}\left(2^{-\nu} m\right) \sum_{l \in \mathbb{Z}^{n}} A(m, l)^{-p(C-A-n-\alpha)}\right)^{1 / p} .
\end{aligned}
$$


The sum over $l \in \mathbb{Z}^{n}$ is bounded by a constant for $C>\frac{n}{p}+n+A+\alpha$. Hence, we get

$$
\begin{aligned}
& 2^{j\left(s-\frac{n}{p}\right)}\left(\sum_{l \in \mathbb{Z}^{n}}\left|\int_{\mathbb{R}^{n}} k_{j l}(y) f^{j}(y) d y\right|^{p} w_{j}^{p}\left(2^{-j} l\right)\right)^{1 / p} \\
& \quad \leq c \sum_{\nu=j+1}^{\infty} 2^{\nu\left(s-\frac{n}{p}\right)} 2^{-(\nu-j) \tilde{\rho}}\left(\sum_{m \in \mathbb{Z}^{n}}\left|\lambda_{\nu m}\right|^{p} w_{\nu}^{p}\left(2^{-\nu} m\right)\right)^{1 / p},
\end{aligned}
$$

where $\tilde{\rho}=A+s-\alpha_{1}-(n / p-n)>0$. Now the result (43) can be obtained from (47), (49) and (50) by standard arguments.

Remark 5. As shown in the proof it is enough to assume

$$
C>A+n\left(1+\frac{1}{\min (1, p)}\right)+\alpha .
$$

We come to the wavelet decomposition theorem. Let us assume, that

$$
\psi_{M} \in C^{k}\left(\mathbb{R}^{n}\right) \quad \text { and } \quad \psi_{F} \in C^{k}\left(\mathbb{R}^{n}\right)
$$

are the real compactly supported Daubechies wavelets from Proposition 5, with

$$
\int_{\mathbb{R}^{n}} x^{\beta} \psi_{M}(x) d x=0 \quad \text { for }|\beta|<k .
$$

By the tensor product procedure (34), we have, that $\left\{\Psi_{G, m}^{\nu}: \nu \in \mathbb{N}_{0}, G \in\right.$ $G^{\nu}$ and $\left.m \in \mathbb{Z}^{n}\right\}$ is an orthonormal basis in $L_{2}\left(\mathbb{R}^{n}\right)$. Before coming to the theorem we clarify the convergence of

$$
\sum_{\nu=0}^{\infty} \sum_{G \in G^{\nu}} \sum_{m \in \mathbb{Z}^{n}} \lambda_{G m}^{\nu} 2^{-\nu \frac{n}{2}} \Psi_{G m}^{\nu} \quad \text { with } \lambda \in \tilde{b}_{p q}^{s, m l o c}(\boldsymbol{w}) .
$$

We say that a series converges unconditionally, if any rearrangement of the series also converges to the same outcome. We know that $\left\{2^{-\nu \frac{n}{2}} \Psi_{G m}^{\nu}\right\}$ are $[k, k, M]$ molecules for every $M>0$ and therefore we have the unconditional convergence of (53) in $\mathcal{S}^{\prime}\left(\mathbb{R}^{n}\right)$ from Lemma 1 with $k>\sigma_{p}-s+\alpha_{1}$. Moreover, the following proof shows the unconditional convergence of (53) in $B_{p q}^{s, m l o c}\left(\mathbb{R}^{n}, \boldsymbol{w}\right)$ for $0<p<\infty$ and $0<q<\infty$. If $0<p<\infty$ and $0<q \leq \infty$ then we have unconditional convergence in $B_{p q}^{\sigma, m l o c}\left(\mathbb{R}^{n}, \boldsymbol{w}\right)$ with $\sigma<s$. For general $0<p, q \leq \infty$ we get the unconditional convergence in 
$B_{p q}^{\sigma, m l o c}\left(\mathbb{R}^{n}, \varrho\right)$ where $\sigma<s$ and $\varrho$ is an admissible weight sequence with $\frac{\varrho_{\nu}(x)}{w_{\nu}(x)} \rightarrow 0$ for $|x| \rightarrow \infty$.

Theorem 4. Let $\boldsymbol{w} \in \mathcal{W}_{\alpha_{1}, \alpha_{2}}^{\alpha}, s \in \mathbb{R}, 0<p, q \leq \infty$ and

$$
k>\max \left(\sigma_{p}-s+\alpha_{1}, s+\alpha_{2}\right)
$$

in (51) and (52). Let $f \in \mathcal{S}^{\prime}\left(\mathbb{R}^{n}\right)$. Then $f \in B_{p q}^{s, m l o c}\left(\mathbb{R}^{n}, \boldsymbol{w}\right)$ if, and only if, it can be represented as

$$
f=\sum_{\nu=0}^{\infty} \sum_{G \in G^{\nu}} \sum_{m \in \mathbb{Z}^{n}} \lambda_{G m}^{\nu} 2^{-\nu \frac{n}{2}} \Psi_{G m}^{\nu} \quad \text { with } \lambda \in \tilde{b}_{p q}^{s, m l o c}(\boldsymbol{w}),
$$

with unconditional convergence in $\mathcal{S}^{\prime}\left(\mathbb{R}^{n}\right)$ and in any space $B_{p q}^{\sigma, m l o c}\left(\mathbb{R}^{n}, \varrho\right)$ with $\sigma<s$ and $\frac{\underline{\rho}_{\nu}(x)}{w_{\nu}(x)} \rightarrow 0$ for $|x| \rightarrow \infty$. The representation (55) is unique, we have

$$
\lambda_{G m}^{\nu}=\lambda_{G m}^{\nu}(f)=2^{\nu \frac{n}{2}}\left\langle f, \Psi_{G m}^{\nu}\right\rangle
$$

and

$$
I: f \mapsto\left\{2^{\nu \frac{n}{2}}\left\langle f, \Psi_{G m}^{\nu}\right\rangle\right\}
$$

is an isomorphic map from $B_{p q}^{s, m l o c}\left(\mathbb{R}^{n}, \boldsymbol{w}\right)$ onto $\tilde{b}_{p q}^{s, m l o c}(\boldsymbol{w})$. Moreover, if in addition $\max (p, q)<\infty$, then $\left\{\Psi_{G m}^{\nu}\right\}$ is in unconditional basis in $B_{p q}^{s, m l o c}\left(\mathbb{R}^{n}, \boldsymbol{w}\right)$.

Proof. First Step: Let $f \in \mathcal{S}^{\prime}\left(\mathbb{R}^{n}\right)$ be given by (55). Then by the support properties we have that $\left\{2^{-\nu \frac{n}{2}} \Psi_{G m}^{\nu}\right\}$ are $[k, k, M]$ molecules for every $M>0$. From Theorem 2 and (54) we obtain $f \in B_{p q}^{s, m l o c}\left(\mathbb{R}^{n}, \boldsymbol{w}\right)$ and

$$
\left\|f\left|B_{p q}^{s, m l o c}\left(\mathbb{R}^{n}, \boldsymbol{w}\right)\|\leq c\| \lambda\right| \tilde{b}_{p q}^{s, m l o c}(\boldsymbol{w})\right\|
$$

with $c>0$ independent of $\lambda \in \tilde{b}_{p q}^{s, m l o c}(\boldsymbol{w})$.

Second Step: Let $f \in B_{p q}^{s, m l o c}\left(\mathbb{R}^{n}, \boldsymbol{w}\right)$ then we can apply Theorem 3 with $k_{\nu m}=2^{\nu \frac{n}{2}} \Psi_{G m}^{\nu}$. Since all conditions on $k_{\nu m}$ are fulfilled by (54) and the compact support of the wavelets we get

$$
\left\|\lambda(f)\left|\tilde{b}_{p q}^{s, m l o c}(\boldsymbol{w})\|\leq c\| f\right| B_{p q}^{s, m l o c}\left(\mathbb{R}^{n}, \boldsymbol{w}\right)\right\| .
$$


Third Step: For $\max (p, q)<\infty$ we get the unconditional convergence of $(55)$ in $B_{p q}^{s, m l o c}\left(\mathbb{R}^{n}, \boldsymbol{w}\right)$ by $(58)$ and the properties of the sequence spaces $\tilde{b}_{p q}^{s, m l o c}(\boldsymbol{w})$.

Let $p<\infty$ and $q=\infty$, then we get the convergence in $B_{p \infty}^{\sigma, m l o c}\left(\mathbb{R}^{n}, \boldsymbol{w}\right)$ for all $\sigma<s$ by using (58) again and Hölder's inequality. To obtain the convergence for $p=\infty$ we have to compensate the behavior at infinity by introducing a weaker weight sequence $\varrho$ with $\frac{\varrho_{\nu}(x)}{w_{\nu}(x)} \rightarrow 0$ for $|x| \rightarrow \infty$. Then we get the unconditional convergence in $B_{\infty q}^{\sigma, m l o c}\left(\mathbb{R}^{n}, \varrho\right)$ with $\sigma<s$ as in the previous case.

A simple example of such a weaker weight sequence is given for every $\varepsilon>0$ by

$$
\varrho_{\nu}(x)=\left(1+2^{\nu}|x|\right)^{-\varepsilon} w_{\nu}(x)
$$

which belongs to $\mathcal{W}_{\alpha_{1}+\varepsilon, \alpha_{2}}^{\alpha+\varepsilon}$.

Fourth Step: We want to prove now the uniqueness of the coefficients. We define

$$
g=\sum_{\nu=0}^{\infty} \sum_{G \in G^{\nu}} \sum_{m \in \mathbb{Z}^{n}} \lambda_{G m}^{\nu} 2^{-\nu \frac{n}{2}} \Psi_{G m}^{\nu}
$$

where $\lambda_{G m}^{\nu}$ is given by (56). We want to show that $g=f$, or

$$
\langle g, \varphi\rangle=\langle f, \varphi\rangle \quad \text { for every } \varphi \in \mathcal{S}\left(\mathbb{R}^{n}\right) .
$$

From the first step we have $g \in B_{p q}^{s, m l o c}\left(\mathbb{R}^{n}, \boldsymbol{w}\right)$. The third step tells us that (61) converges at least in $B_{p q}^{\sigma, m l o c}\left(\mathbb{R}^{n}, \varrho\right)$ for all $\sigma<s$ and $\varrho$ is given by (60) for some $\varepsilon>0$. Since $k>\sigma_{p}-s+\alpha_{1}$ we can find $\sigma<s$ and $\varepsilon>0$ such that $\Psi_{G^{\prime} m^{\prime}}^{\nu^{\prime}}$ still belongs to the dual space $\left(B_{p q}^{\sigma, m l o c}\left(\mathbb{R}^{n}, \varrho\right)\right)^{\prime}$ (that means $\left.k>\sigma_{p}-\sigma+\alpha_{1}+\varepsilon\right)$. Because of the convergence in $B_{p q}^{\sigma, m l o c}\left(\mathbb{R}^{n}, \varrho\right)$ ,the dual pairing and the orthonormality of $\left\{\Psi_{G m}^{\nu}\right\}$ in $L_{2}\left(\mathbb{R}^{n}\right)$ we get

$$
\left\langle g, \Psi_{G^{\prime} m^{\prime}}^{\nu^{\prime}}\right\rangle=\left\langle f, \Psi_{G^{\prime} m^{\prime}}^{\nu^{\prime}}\right\rangle
$$

This holds also for finite linear combinations of $\Psi_{G^{\prime} m^{\prime}}^{\nu^{\prime}}$. For a function $\varphi \in \mathcal{S}\left(\mathbb{R}^{n}\right)$ we have the unique $L_{2}\left(\mathbb{R}^{n}\right)$ representation

$$
\varphi=\sum_{\nu, G, m} 2^{-j \frac{n}{2}}\left\langle\varphi, \Psi_{G m}^{\nu}\right\rangle \Psi_{G m}^{\nu} .
$$


Since $\mathcal{S}\left(\mathbb{R}^{n}\right)$ is a subspace in every Besov space considered this representation converges in $\left(B_{p, q}^{\sigma, m l o c}\left(\mathbb{R}^{n}, \boldsymbol{w}\right)\right)^{\prime}$ and we get by $(62)$

$$
\langle g, \varphi\rangle=\langle f, \varphi\rangle \text {. }
$$

Final Step: Hence, $f \in \mathcal{S}^{\prime}\left(\mathbb{R}^{n}\right)$ belongs to $B_{p q}^{s, m l o c}\left(\mathbb{R}^{n}, \boldsymbol{w}\right)$ if and only if, it can be represented by (55). This representation is unique with coefficients (56). By (58), (61), with $g=f$, and (59) it follows

$$
\left\|\lambda(f)\left|\tilde{b}_{p q}^{s, m l o c}(\boldsymbol{w})\|\sim\| f\right| B_{p q}^{s, m l o c}\left(\mathbb{R}^{n}, \boldsymbol{w}\right)\right\| .
$$

Hence $I$ in (57) is an isomorphic map from $B_{p q}^{s, m l o c}\left(\mathbb{R}^{n}, \boldsymbol{w}\right)$ into $\tilde{b}_{p q}^{s, m l o c}(\boldsymbol{w})$. It remains to prove that this map is onto. Let $\lambda \in \tilde{b}_{p q}^{s, m l o c}(\boldsymbol{w})$. Then it follows by the above considerations that

$$
f=\sum_{\nu, G, m} \lambda_{G m}^{\nu} 2^{-\nu \frac{n}{2}} \Psi_{G m}^{\nu} \quad \in B_{p q}^{s, m l o c}\left(\mathbb{R}^{n}, \boldsymbol{w}\right) .
$$

By the same reasoning as in the fourth step this representation is unique and $\lambda_{G m}^{\nu}=\lambda_{G m}^{\nu}(f)$. This proves that $I$ is a map onto.

To prove the wavelet decomposition with Daubechies wavelets we used only the atomic decomposition theorem. Now, we present a wavelet decomposition theorem with the help of Meyer wavelets, described in Proposition 5. We have $\psi_{M}, \psi_{F} \in \mathcal{S}\left(\mathbb{R}^{n}\right)$ and we have infinitely many moment conditions on $\psi_{M}$. But we lose the compact support property for the wavelets. Here we need to use our molecular decomposition. The proof is the same as in Theorem 4. We use again our wavelets once as molecules and once as kernels from Definition 8 where the technicalities turn out to be easier because $A, B, C$ are infinite.

Theorem 5. Let $\left\{\Psi_{G m}^{\nu}\right\}$ be the Meyer wavelets according to Proposition 5. Further, let $\boldsymbol{w} \in \mathcal{W}_{\alpha_{1}, \alpha_{2}}^{\alpha}, s \in \mathbb{R}, 0<p, q \leq \infty$ and let $f \in \mathcal{S}^{\prime}\left(\mathbb{R}^{n}\right)$. Then $f \in B_{p q}^{s, m l o c}\left(\mathbb{R}^{n}, \boldsymbol{w}\right)$ if, and only if, it can be represented as

$$
f=\sum_{\nu=0}^{\infty} \sum_{G \in G^{\nu}} \sum_{m \in \mathbb{Z}^{n}} \lambda_{G m}^{\nu} 2^{-\nu \frac{n}{2}} \Psi_{G m}^{\nu} \quad \text { with } \lambda \in \tilde{b}_{p q}^{s, m l o c}(\boldsymbol{w})
$$

with unconditional convergence in $\mathcal{S}^{\prime}\left(\mathbb{R}^{n}\right)$ and in any space $B_{p q}^{\sigma, m l o c}\left(\mathbb{R}^{n}, \varrho\right)$ with $\sigma<s$ and $\frac{\varrho_{\nu}(x)}{w_{\nu}(x)} \rightarrow 0$ for $|x| \rightarrow \infty$. The representation (63) is unique,

$$
\lambda_{G m}^{\nu}=\lambda_{G m}^{\nu}(f)=2^{\nu \frac{n}{2}}\left\langle f, \Psi_{G m}^{\nu}\right\rangle
$$


and

$$
I: f \mapsto\left\{2^{\nu \frac{n}{2}}\left\langle f, \Psi_{G m}^{\nu}\right\rangle\right\}
$$

is an isomorphic map from $B_{p q}^{s, m l o c}\left(\mathbb{R}^{n}, \boldsymbol{w}\right)$ onto $\tilde{b}_{p q}^{s, m l o c}(\boldsymbol{w})$. Moreover, if in addition $\max (p, q)<\infty$ then $\left\{\Psi_{G m}^{\nu}\right\}$ is in unconditional basis in $B_{p q}^{s, m l o c}\left(\mathbb{R}^{n}, \boldsymbol{w}\right)$.

Remark 6. The wavelet characterization of $B_{p q}^{s, m l o c}\left(\mathbb{R}^{n}, \boldsymbol{w}\right)$ is not restricted to the two wavelet systems presented in Proposition 5. The proof of Theorem 4 also applies (at least for $\max (p, q)<\infty$ due to duality) to all wavelet systems $\left\{\Psi_{G m}^{\nu}\right\}$ which satisfy that $2^{-\nu \frac{n}{2}}\left\{\Psi_{G m}^{\nu}\right\}$ are $[K, K, M]$ molecules with

$$
K>\max \left(\sigma_{p}-s+\alpha_{1}, s+\alpha_{2}\right) \text { and } M>K+n \max (2,1+1 / p)+2 \alpha,
$$

where the condition on $M$ is not sharp. The proofs can easily be extended to biorthogonal wavelet bases (see [11] for details).

5.4 Wavelet decomposition of $\mathbf{B}_{\mathbf{p q}}^{\mathbf{s}, \mathbf{s}^{\prime}}\left(\mathbb{R}^{\mathbf{n}}, \mathbf{U}\right)$. For a bounded subset $U \subset \mathbb{R}^{n}$ and $s^{\prime} \in \mathbb{R}$ we have the 2 -microlocal weights

$$
w_{\nu}(x)=\left(1+2^{\nu} \operatorname{dist}(x, U)\right)^{s^{\prime}} .
$$

We know that $\boldsymbol{w} \in \mathcal{W}_{\alpha_{1}, \alpha_{2}}^{\alpha}$ for $\left|s^{\prime}\right| \leq \alpha$ and $-\min \left(0, s^{\prime}\right) \leq \alpha_{1}, \max \left(0, s^{\prime}\right) \leq$ $\alpha_{2}$. The spaces $B_{p q}^{s, s^{\prime}}\left(\mathbb{R}^{n}, U\right)$ are defined as the spaces $B_{p q}^{s, m l o c}\left(\mathbb{R}^{n}, \boldsymbol{w}\right)$ with the special weight sequence $\boldsymbol{w}$ defined in (65). The corresponding sequence spaces are defined by the norm

$$
\begin{aligned}
& \left\|\lambda \mid \tilde{b}_{p q}^{s, s^{\prime}}(U)\right\| \\
& =\left(\sum_{\nu=0}^{\infty} 2^{\nu(s-n / p) q} \sum_{G \in G^{\nu}}\left(\sum_{m \in \mathbb{Z}^{n}}\left|\lambda_{G m}^{\nu}\right|^{p}\left(1+2^{\nu} \operatorname{dist}\left(2^{-\nu} m, U\right)\right)^{p s^{\prime}}\right)^{q / p}\right)^{1 / q} .
\end{aligned}
$$

Now, we can adapt everything from Theorem 4 and a wavelet decomposition for the 2-microlocal Besov spaces with respect to the Daubechies wavelets follows.

Corollary 2. Let $U \subset \mathbb{R}^{n}$ bounded, $s^{\prime} \in \mathbb{R}$ and $\boldsymbol{w}$ as in (65). Further, let $s \in \mathbb{R}, 0<p, q \leq \infty$ and

$$
k>\max \left(\sigma_{p}-s-\min \left(0, s^{\prime}\right), s+\max \left(0, s^{\prime}\right)\right)
$$


in (51) and (52). Let $f \in \mathcal{S}^{\prime}\left(\mathbb{R}^{n}\right)$. Then $f \in B_{p q}^{s, s^{\prime}}\left(\mathbb{R}^{n}, U\right)$ if, and only if, it can be represented as

$$
f=\sum_{\nu=0}^{\infty} \sum_{G \in G^{\nu}} \sum_{m \in \mathbb{Z}^{n}} \lambda_{G m}^{\nu} 2^{-\nu \frac{n}{2}} \Psi_{G m}^{\nu} \quad \text { with } \lambda \in \tilde{b}_{p q}^{s, s^{\prime}}(U)
$$

with unconditional convergence in $\mathcal{S}^{\prime}\left(\mathbb{R}^{n}\right)$ and in any space $B_{p q}^{t, t^{\prime}}\left(\mathbb{R}^{n}, U\right)$ with $t<s$ and $t^{\prime}<s^{\prime}$. The representation (67) is unique,

$$
\lambda_{G m}^{\nu}=\lambda_{G m}^{\nu}(f)=2^{\nu \frac{n}{2}}\left\langle f, \Psi_{G m}^{\nu}\right\rangle
$$

and

$$
I: f \mapsto\left\{2^{\nu \frac{n}{2}}\left\langle f, \Psi_{G m}^{\nu}\right\rangle\right\}
$$

is an isomorphic map from $B_{p q}^{s, s^{\prime}}\left(\mathbb{R}^{n}, U\right)$ onto $\tilde{b}_{p q}^{s, s^{\prime}}(U)$. Moreover, if in addition $\max (p, q)<\infty$, then $\left\{\Psi_{G m}^{\nu}\right\}$ is in unconditional basis in $B_{p q}^{s, s^{\prime}}\left(\mathbb{R}^{n}, U\right)$.

Let us say a few words about the convergence of (67). As in Theorem 4 we have unconditional convergence in $B_{p q}^{s, s^{\prime}}\left(\mathbb{R}^{n}, U\right)$ for $\max (p, q)<\infty$ and arbitrary $U \in \mathbb{R}^{n}$, not necessarily bounded. For $0<p<\infty$ and $0<q \leq \infty$ we have unconditional convergence in $B_{p q}^{t, s^{\prime}}\left(\mathbb{R}^{n}, U\right)$ with $s>t$ and also arbitrary $U$. Only in the case of $p=\infty$ we need as an additional assumption that $U \subset \mathbb{R}^{n}$ has to be bounded to get the unconditional convergence in $B_{p q}^{t, t^{\prime}}\left(\mathbb{R}^{n}, U\right)$ for all $s>t$ and $s^{\prime}>t^{\prime}$.

Remark 7. For $p \geq 1$ we have $\sigma_{p}=0$ in condition (66). Now we can rewrite this condition as $k>\max \left(|s|,\left|s+s^{\prime}\right|\right)$. This is almost the same condition $k>\max \left(\max \left(s, s+s^{\prime}\right), \max \left(-(s+n),-\left(s+s^{\prime}\right)\right)\right)$ used in [14, p.64, p.67] in connection with the orthonormal Daubechies wavelets.

According to Remark 6 we can also give a characterization of $B_{p q}^{s, s^{\prime}}\left(\mathbb{R}^{n}, U\right)$ by other wavelet bases $\left\{\Psi_{G m}^{\nu}\right\}$ (for example the Meyer wavelets), where $\left\{\Psi_{G m}^{\nu}\right\}$ are $[K, K, M]$ molecules with the condition (64) on $K$ and $M$.

\section{Pseudodifferential Operators}

In this section we show that the 2-microlocal spaces $B_{p q}^{s, m l o c}\left(\mathbb{R}^{n}, \boldsymbol{w}\right)$ are invariant under the action of classical pseudodifferential operators of order 0 . The corresponding results have already been shown in [8] for $C_{x_{0}}^{s, s^{\prime}}\left(\mathbb{R}^{n}\right)$ and in [1] for $F_{p, q}^{s, s^{\prime}}\left(\mathbb{R}^{n}, x_{0}\right), 1 \leq p, q \leq \infty$. 
As in [8] we introduce operators $T$ belonging to the class $O p\left(M^{\gamma}\right)$ of Calderón-Zygmund operators, which were introduced in [13].

A linear and continuous operator $T: \mathcal{S}\left(\mathbb{R}^{n}\right) \rightarrow \mathcal{S}^{\prime}\left(\mathbb{R}^{n}\right)$ possesses by the Schwartz kernel Theorem a distribution kernel $K$ in $\mathcal{S}^{\prime}\left(\mathbb{R}^{n} \times \mathbb{R}^{n}\right)$ such that $\langle T f, g\rangle=\langle K, f \otimes g\rangle$ for all $f, g \in \mathcal{S}\left(\mathbb{R}^{n}\right)$. This kernel $K$ belongs to the class $O^{\gamma}$ for $\gamma>0$, if its restriction to the set $\left\{(x, y) \in \mathbb{R}^{n} \times \mathbb{R}^{n}: x \neq y\right\}$ is continuous and satisfies

$$
\left|D^{\beta} K(x, y)\right| \leq c|x-y|^{-n-|\beta|} \text { for }|\beta|<\gamma
$$

and, with $\gamma=m+r$, where $m \in \mathbb{N}_{0}$ and $r \in(0,1]$

$$
\begin{array}{ll}
\left|D^{\beta} K(x, y)-D^{\beta} K\left(x^{\prime}, y\right)\right| \leq c \frac{\left|x-x^{\prime}\right|^{r}}{|x-y|^{n+\gamma}} \quad \text { for }\left|x-x^{\prime}\right| \leq \frac{1}{2}|x-y| \\
\left|D^{\beta} K(x, y)-D^{\beta} K\left(x, y^{\prime}\right)\right| \leq c \frac{\left|y-y^{\prime}\right|^{r}}{|x-y|^{n+\gamma}} \quad \text { for }\left|y-y^{\prime}\right| \leq \frac{1}{2}|x-y|
\end{array}
$$

for $|\beta|=m$. Moreover, $T$ has to be bounded on $L_{2}\left(\mathbb{R}^{n}\right)$ and $T\left(X^{\beta}\right)=$ $T^{*}\left(X^{\beta}\right)=0$ for $|\beta| \leq \gamma$ (see [13] for definition of $T\left(X^{\beta}\right)$ ). Then $O p\left(M^{\gamma}\right)=\bigcup_{\gamma^{\prime}>\gamma} O^{\gamma^{\prime}}$. The class $O p\left(M^{\gamma}\right)$ can be characterized by decay conditions on the terms of the matrix $\left(\left\langle T \Psi_{\lambda}, \Psi_{\lambda^{\prime}}\right\rangle\right)$ with respect to a wavelet basis $\left\{\Psi_{\lambda}\right\}$ where $\Psi_{\lambda}=\Psi_{G m}^{\nu}$ (see [13, Chapter 8]).

Proposition 6. An operator $T$ belongs to $O p\left(M^{\gamma}\right)$ if and only if for some $\gamma^{\prime}>\gamma$ its matrix coefficients satisfy

$$
\left|\left\langle T \Psi_{G m}^{\nu}, \Psi_{G l}^{j}\right\rangle\right| \leq c \frac{2^{-|j-\nu|\left(\gamma^{\prime}+n / 2\right)}}{1+(j-\nu)^{2}}\left(1+\frac{\left|2^{-\nu} m-2^{-j} l\right|}{2^{-\nu}+2^{-j}}\right)^{-n-\gamma^{\prime}} .
$$

The condition in (68) can be reformulated as

$$
\begin{aligned}
& 2^{(\nu-j) n / 2} \mid\left\langle T \Psi_{G m}^{\nu}, \Psi_{G l}^{j}\right\rangle \mid \\
&(69) \quad \leq c\left\{\begin{array}{ll}
2^{-(\nu-j) \gamma^{\prime}}\left(1+2^{j}\left|2^{-j} l-2^{-\nu} m\right|\right)^{-n-\gamma^{\prime}}, & \text { for } \nu \geq j \\
2^{-(j-\nu)\left(n+\gamma^{\prime}\right)}\left(1+2^{\nu}\left|2^{-j} l-2^{-\nu} m\right|\right)^{-n-\gamma^{\prime}}, & \text { for } \nu \leq j
\end{array} .\right.
\end{aligned}
$$

That means that our matrix is almost diagonal (in the meaning of [6]) and we can easily adopt the proof of Theorem 3 with (69) instead of the Lemma 5.

Theorem 6. Let $\boldsymbol{w} \in \mathcal{W}_{\alpha_{1}, \alpha_{2}}^{\alpha}, 0<p, q \leq \infty$ and $s \in \mathbb{R}$. If $f \in$ $B_{p q}^{s, m l o c}\left(\mathbb{R}^{n}, \boldsymbol{w}\right)$ and $T \in O p\left(M^{\gamma}\right)$ with $\gamma>\max \left(s+\alpha_{2}, \sigma_{p}-s+\alpha_{1}, \sigma_{p}+\alpha\right)$ then $T f \in B_{p q}^{s, m l o c}\left(\mathbb{R}^{n}, \boldsymbol{w}\right)$. 
If we keep the last Theorem in mind, we get the following interpretation. The position of points of regularity of a function is essentially preserved under the action of singular integral operators, such as the Hilbert transform or the Riesz transforms $R_{j}=-i \frac{\partial}{\partial x_{j}}(-\Delta)^{-1 / 2}$. Moreover, since every classical pseudodifferential operator belonging to $S_{1,0}^{0}$ is a sum of an operator in $O p\left(M^{\gamma}\right)$ and a regularizing operator ([13, Chapter 7]), we conclude that the 2-microlocal spaces $B_{p q}^{s, m l o c}\left(\mathbb{R}^{n}, \boldsymbol{w}\right)$ are invariant under the action of pseudodifferential operators out of $S_{1,0}^{0}$. We can state now regularity result for solutions of elliptic partial differential equations. From Calderón and Zygmund [3] we know, that the inverse of an elliptic operator is a product of a fractional integration and a pseudodifferential operator of order 0 . The latter one is a sum of an operator in $O p\left(M^{\gamma}\right)$ and a regularizing operator and the first one is a lift operator (Proposition 2). Using this fact together with the last Theorem, we obtain the following assertion.

Theorem 7. Let $\boldsymbol{w} \in \mathcal{W}_{\alpha_{1}, \alpha_{2}}^{\alpha}, 0<p, q \leq \infty$ and $s \in \mathbb{R}$. Let $\Lambda$ be an elliptic partial differential operator of order $m$, with smooth coefficients. If $\Lambda f=g$ and $g \in B_{p q}^{s, m l o c}\left(\mathbb{R}^{n}, \boldsymbol{w}\right)$, then $f \in B_{p q}^{s+m, m l o c}\left(\mathbb{R}^{n}, \boldsymbol{w}\right)$.

\section{Appendix}

Here we present the more technical proofs of the Lemmas.

Proof of Lemma 1. We have to prove that the limit

$$
\lim _{r \rightarrow \infty} \sum_{\nu=0}^{r} \sum_{m \in \mathbb{Z}^{n}} \lambda_{\nu m} \mu_{\nu m}(x) \quad \text { exists in } \mathcal{S}^{\prime}\left(\mathbb{R}^{n}\right) .
$$

For $\varphi \in \mathcal{S}\left(\mathbb{R}^{n}\right)$ we get from the moment conditions (17) for fixed $\nu \in \mathbb{N}_{0}$

$$
\int_{\mathbb{R}^{n}} \sum_{m \in \mathbb{Z}^{n}} \lambda_{\nu m} \mu_{\nu m}(y) \varphi(y) d y
$$

$$
\begin{aligned}
=\int_{\mathbb{R}^{n}} \sum_{m \in \mathbb{Z}^{n}} \lambda_{\nu m} \mu_{\nu m}(y) w_{\nu}(y) \times \\
\quad \times\left(\varphi(y)-\sum_{|\beta|<L} \frac{D^{\beta} \varphi\left(2^{-\nu} m\right)}{\beta !}\left(y-2^{-\nu} m\right)^{\beta}\right) w_{\nu}^{-1}(y) \frac{\langle y\rangle^{\kappa}}{\langle y\rangle^{\kappa}} d y,
\end{aligned}
$$


where $\kappa>0$ will be specified later on. We use Taylor expansion of $\varphi$ up to the order $L$ and get with $\xi$ on the line segment joining $y$ and $2^{-\nu} m$

$$
\varphi(y)=\sum_{|\beta|<L} \frac{D^{\beta} \varphi\left(2^{-\nu} m\right)}{\beta !}\left(y-2^{-\nu} m\right)^{\beta}+\sum_{|\beta|=L} \frac{D^{\beta} \varphi(\xi)}{\beta !}\left(y-2^{-\nu} m\right)^{\beta} .
$$

Using the properties of the weight sequence and $\langle y\rangle^{\kappa} \leq\left\langle y-2^{-\nu} m\right\rangle^{\kappa}\langle\xi\rangle^{\kappa}$, we can estimate (71) by

$$
\begin{aligned}
&\left|\mu_{\nu m}(y)\right| \varphi(y)-\sum_{|\beta|<L} \frac{D^{\beta} \varphi\left(2^{-\nu} m\right)}{\beta !}\left(y-2^{-\nu} m\right)^{\beta} \mid w_{\nu}^{-1}(y) \frac{\langle y\rangle^{\kappa}}{\langle y\rangle^{\kappa}} \\
& \leq c 2^{-\nu\left(L-\alpha_{1}\right)}\left(1+2^{\nu}\left|y-2^{-\nu} m\right|\right)^{-M} \times \\
& \times \sum_{|\beta|=L} \frac{\left|D^{\beta} \varphi(\xi)\right|}{\beta !}\left|y-2^{-\nu} m\right|^{L} 2^{\nu L} w_{0}^{-1}(y) \frac{\langle\xi\rangle^{\kappa}\left\langle y-2^{-\nu} m\right\rangle^{\kappa}}{\langle y\rangle^{\kappa}} \\
& \leq c^{\prime} 2^{-\nu\left(L-\alpha_{1}\right)}\left(1+2^{\nu}\left|y-2^{-\nu} m\right|\right)^{L+\kappa-M}\langle y\rangle^{\alpha-\kappa}\|\varphi\|_{\kappa, L} .
\end{aligned}
$$

Hence, we derive from (71)

$$
\begin{aligned}
& \left|\int_{\mathbb{R}^{n}} \sum_{m \in \mathbb{Z}^{n}} \lambda_{\nu m} \mu_{\nu m}(y) \varphi(y) d y\right| \\
& \leq c 2^{-\nu\left(L-\alpha_{1}\right)}\|\varphi\|_{\kappa, L} \int_{\mathbb{R}^{n}} \sum_{m \in \mathbb{Z}^{n}}\left|\lambda_{\nu m}\right| w_{\nu}(y)\left(1+2^{\nu}\left|y-2^{-\nu} m\right|\right)^{L+\kappa-M}\langle y\rangle^{\alpha-\kappa} d y .
\end{aligned}
$$

Now, let us suppose that $p \geq 1$ then we get applying Hölder's inequality on the integral in (72) with $\kappa>\frac{n}{p^{\prime}}+\alpha \geq n+\alpha$

$$
\begin{aligned}
\int_{\mathbb{R}^{n}} \sum_{m \in \mathbb{Z}^{n}} \mid & \lambda_{\nu m} \mid w_{\nu}(y)\left(1+2^{\nu}\left|x-2^{-\nu} m\right|\right)^{L+\kappa-M}\langle y\rangle^{\alpha-\kappa} d y \\
\leq c 2^{-\nu\left(L+s-\alpha_{1}\right)} 2^{\nu s} \| & \sum_{m \in \mathbb{Z}^{n}}\left|\lambda_{\nu m}\right|\left(1+2^{\nu}\left|x-2^{-\nu} m\right|\right)^{L+\kappa-M} \mid L_{p}\left(\mathbb{R}^{n}, w_{\nu}\right) \| .
\end{aligned}
$$


By choosing $M$ large enough $(M>L+2 n+2 \alpha)$ and using Lemma 4 with $j=\nu$ we get

$$
\begin{aligned}
& \left|\int_{\mathbb{R}^{n}} \sum_{m \in \mathbb{Z}^{n}} \lambda_{\nu m} \mu_{\nu m}(y) \varphi(y) d y\right| \\
& \quad \leq c 2^{-\nu\left(L+s-\alpha_{1}\right)} 2^{\nu\left(s-\frac{n}{p}\right)}\left(\sum_{m \in \mathbb{Z}^{n}}\left|\lambda_{\nu m}\right|^{p} w_{\nu}^{p}\left(2^{-\nu} m\right)\right)^{1 / p}\|\varphi\|_{\kappa, L} .
\end{aligned}
$$

Since $L>\sigma_{p}-s+\alpha_{1}=-s+\alpha_{1}$ and $\lambda \in b_{p q}^{s, \boldsymbol{w}} \hookrightarrow b_{p \infty}^{s, \boldsymbol{w}}$, the convergence of $(70)$ in $\mathcal{S}^{\prime}\left(\mathbb{R}^{n}\right)$ follows. If $p<1$, we get analogously by choosing $\kappa=\alpha$, $M>L+n+2 \alpha$ and using Hölder's inequality and the weight property $w_{\nu}(y) \leq C w_{\nu}\left(2^{-\nu} m\right)\left(1+2^{\nu}\left|y-2^{-\nu} m\right|\right)^{\alpha}$

$$
\begin{aligned}
& \left|\int_{\mathbb{R}^{n}} \sum_{m \in \mathbb{Z}^{n}} \lambda_{\nu m} \mu_{\nu m}(y) \varphi(y) d y\right|^{p} \\
& \leq c 2^{-\nu\left(L+s-\alpha_{1}\right)} 2^{\nu s} \sum_{m \in \mathbb{Z}^{n}}\left|\lambda_{\nu m}\right|^{p} w_{\nu}^{p}\left(2^{-\nu} m\right) \\
& \quad \times \int_{\mathbb{R}^{n}}\left(1+2^{\nu}\left|y-2^{-\nu} m\right|\right)^{L+2 \alpha-M} d y\|\varphi\|_{\alpha, L} \\
& \leq c^{\prime} 2^{-\nu\left(L+s+n-\frac{n}{p}-\alpha_{1}\right)} 2^{\nu\left(s-\frac{n}{p}\right)} \sum_{m \in \mathbb{Z}^{n}}\left|\lambda_{\nu m}\right|^{p} w_{\nu}^{p}\left(2^{-\nu} m\right)\|\varphi\|_{\alpha, L} .
\end{aligned}
$$

Finally, using $\ell_{p} \hookrightarrow \ell_{1}, L>\sigma_{p}-s+\alpha_{1}=n\left(\frac{1}{p}-1\right)-s+\alpha_{1}$ and $\lambda \in b_{p q}^{s, \boldsymbol{w}} \hookrightarrow b_{p \infty}^{s, \boldsymbol{w}}$ we get the $\mathcal{S}^{\prime}\left(\mathbb{R}^{n}\right)$ convergence of (70).

Proof of Lemma 4. First Step: We treat the case $j \leq \nu$. We decompose $\mathbb{R}^{n}$ into cubes $Q_{\nu l}$ and get

$$
\begin{aligned}
\left\|F \mid L_{p}\left(\mathbb{R}^{n}, w_{j}\right)\right\|^{p} & =\int_{\mathbb{R}^{n}} w_{j}^{p}(x)\left|\sum_{m \in \mathbb{Z}^{n}} \lambda_{\nu m} f_{\nu m}(x)\right|^{p} d x \\
& \leq c \sum_{l \in \mathbb{Z}^{n}} \int_{Q_{\nu l}}\left|\sum_{m \in \mathbb{Z}^{n}} \lambda_{\nu m} w_{j}(x)\left(1+2^{j}\left|x-2^{-\nu} m\right|\right)^{-R}\right|^{p} d x .
\end{aligned}
$$

Now, we use

$$
w_{j}(x) \leq \mathrm{C} 2^{\alpha_{1}(\nu-j)} w_{\nu}\left(2^{-\nu} m\right)\left(1+2^{j}\left|x-2^{-\nu} m\right|\right)^{\alpha}
$$

and that $x \in Q_{\nu l}$ (that means $0 \leq\left|x-2^{-\nu} l\right| \leq c 2^{-\nu}$ ). This leads to 


$$
\begin{aligned}
& \left\|F \mid L_{p}\left(\mathbb{R}^{n}, w_{j}\right)\right\|^{p} \\
& \quad \leq c 2^{\alpha_{1} p(\nu-j)} \sum_{l \in \mathbb{Z}^{n}} \int_{Q_{\nu l}}\left|\sum_{m \in \mathbb{Z}^{n}} \lambda_{\nu m} w_{\nu}\left(2^{-\nu} m\right)\left(1+2^{j-\nu}|l-m|\right)^{-R+\alpha}\right|^{p} d x \\
& \quad \leq c 2^{\alpha_{1} p(\nu-j)} 2^{-\nu n} \sum_{l \in \mathbb{Z}^{n}}\left(\sum_{m \in \mathbb{Z}^{n}}\left|\lambda_{\nu m}\right| w_{\nu}\left(2^{-\nu} m\right)\left(1+2^{j-\nu}|l-m|\right)^{-R+\alpha}\right)^{p}
\end{aligned}
$$

and Young's inequality gives us

$$
\leq c 2^{\alpha_{1} p(\nu-j)} 2^{-\nu n}\left(\sum_{m \in \mathbb{Z}^{n}}\left|\lambda_{\nu m}\right|^{p} w_{\nu}^{p}\left(2^{-\nu} m\right)\right)\left(\sum_{l \in \mathbb{Z}^{n}}\left(1+2^{j-\nu}|l|\right)^{-R+\alpha}\right)^{p} .
$$

Finally, we have to estimate the last sum in (73). Splitting the sum we obtain

$$
\begin{aligned}
\sum_{l \in \mathbb{Z}^{n}}\left(1+2^{j-\nu}|l|\right)^{-R+\alpha} & =\sum_{|l| \leq 2^{\nu-j}}\left(1+2^{j-\nu}|l|\right)^{-R+\alpha}+\sum_{|l|>2^{\nu-j}}\left(1+2^{j-\nu}|l|\right)^{-R+\alpha} \\
& \leq c 2^{(\nu-j) n}+\int_{|y|>2^{\nu-j}}\left(1+2^{j-\nu}|y|\right)^{-R+\alpha} d y \\
& \leq c^{\prime} 2^{(\nu-j) n}, \quad \text { for } R>\alpha+n .
\end{aligned}
$$

Putting this into (73) and taking the $1 / p$ power, the first part of the lemma is proved.

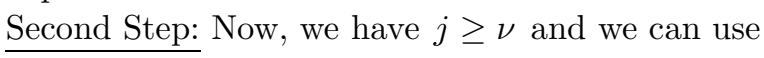

$$
f_{\nu m}(x)=\left(1+2^{j}\left|x-2^{-\nu} m\right|\right)^{-R} \leq c\left(1+2^{\nu}\left|x-2^{-\nu} m\right|\right)^{-R}
$$

and

$$
w_{j}(x) \leq \mathrm{C} 2^{\alpha_{2}(j-\nu)} w_{\nu}\left(2^{-\nu} m\right)\left(1+2^{\nu}\left|x-2^{-\nu} m\right|\right)^{\alpha} .
$$

The same splitting of the integral in dyadic cubes as in the first step together with the above inequalities lead to the second case.

Proof of Lemma 5. To prove the case $\nu \geq j$ one can modify the steps in [5, Lemma 3.3]. We only need the moment conditions on $\mu_{\nu m}$ and a sufficiently strong decay of the derivatives of $k_{j, l}$. The proof for $\nu \geq j$ follows easily by interchanging the roles of $k_{j l}$ and $\mu_{\nu m}$. 
Proof of Lemma 6. Here we only give the proof for $\nu \geq j$ because the other case follows by similar arguments. For $k \in \mathbb{Z}^{n}$ we introduce the quantity

$$
\ell_{\nu}^{j}(k)=\left\{m \in \mathbb{Z}^{n}: Q_{j k} \cap Q_{\nu m} \neq \emptyset\right\}=\left\{m \in \mathbb{Z}^{n}: Q_{\nu m} \subseteq Q_{j k}\right\} .
$$

Then we get for $m \in \ell_{\nu}^{j}(k)$

$$
(1+|l-k|) \leq c\left(1+2^{j}\left|2^{-j} l-2^{-\nu} m\right|\right) .
$$

Estimate (74) and Minkowski's inequality yield

$$
\begin{aligned}
&\left(\sum_{l \in \mathbb{Z}^{n}}\left(\sum_{m \in \mathbb{Z}^{n}}\left|b_{m}\right|\left(1+2^{j}\left|2^{-j} l-2^{-\nu} m\right|\right)^{-R}\right)^{p}\right)^{1 / p} \\
&=\left(\sum_{l \in \mathbb{Z}^{n}}\left(\sum_{k \in \mathbb{Z}^{n}} \sum_{m \in \ell_{\nu}^{j}(k)}\left|b_{m}\right|\left(1+2^{j}\left|2^{-j} l-2^{-\nu} m\right|\right)^{-R}\right)^{p}\right)^{1 / p} \\
& \leq c\left(\sum_{l \in \mathbb{Z}^{n}}\left(\sum_{k \in \mathbb{Z}^{n}}(1+|k-l|)^{-R} \sum_{m \in \ell_{\nu}^{j}(k)}\left|b_{m}\right|\right)^{p}\right)^{1 / p} \\
&=c\left(\sum_{l \in \mathbb{Z}^{n}}\left(\sum_{u \in \mathbb{Z}^{n}}(1+|u|)^{-R} \sum_{m \in \ell_{\nu}^{j}(u+l)}\left|b_{m}\right|\right)^{p}\right)^{1 / p} \\
& \leq c \sum_{u \in \mathbb{Z}^{n}}(1+|u|)^{-R}\left(\sum_{l \in \mathbb{Z}^{n}}\left(\sum_{m \in \ell_{\nu}^{j}(u+l)}\left|b_{m}\right|\right)^{p}\right)^{1 / p} .
\end{aligned}
$$

Finally Hölder's inequality and $\operatorname{card} \ell_{\nu}^{j}(u+l) \sim 2^{(\nu-j) n}$ imply

$$
\begin{aligned}
\left(\sum _ { l \in \mathbb { Z } ^ { n } } \left(\sum_{m \in \mathbb{Z}^{n}}\left|b_{m}\right|(1+\right.\right. & \left.\left.\left.2^{j}\left|2^{-j} l-2^{-\nu} m\right|\right)^{-R}\right)^{p}\right)^{1 / p} \\
& \leq c 2^{(\nu-j) \frac{n}{p^{\prime}}} \sum_{u \in \mathbb{Z}^{n}}(1+|u|)^{-R}\left(\sum_{l \in \mathbb{Z}^{n}} \sum_{m \in \ell_{\nu}^{j}(u+l)}\left|b_{m}\right|^{p}\right)^{1 / p} \\
& \leq c^{\prime} 2^{(\nu-j) \frac{n}{p^{\prime}}}\left(\sum_{m \in \mathbb{Z}^{n}}\left|b_{m}\right|^{p}\right)^{1 / p},
\end{aligned}
$$


where the last inequality is due to $\operatorname{card}\left\{l \in \mathbb{Z}^{n}: Q_{\nu m} \subseteq Q_{j l}+2^{-j} u\right\} \sim 1$ and $R>n$.

\section{References}

[1] P. Andersson, Two-microlocal spaces, local norms and weighted spaces, Paper 2 in PhD Thesis, University of Göteborg (1997), 35-58.

[2] J.M. Bony, Second microlocalization and propagation of singularities for semi-linear hyperbolic equations, Taniguchi Symp. HERT. Katata (1984), 11-49.

[3] A.P. Calderón and A. Zygmund, Singular integral operators and differential equations, Amer. J. Math. (1957), 901-921.

[4] D.E. Edmunds and H. Triebel, Function Spaces, Entropy Numbers, Differential Operators, Cambridge Univ. Press, 1996.

[5] M. Frazier and B. Jawerth, Decomposition of Besov spaces, Indiana Univ. Math. J., 34 (1985), 777-799.

[6] M. Frazier and B. Jawerth, A discrete transform and decompositions of distribution spaces, J. Funct. Anal., 93 (1990), 34-170.

[7] M. Frazier, B. Jawerth and G. Weiss, Littlewood-Paley Theory and the Study of Function Spaces, CBMS Reg. Conf. Ser. Math., AMS, 79, 1991.

[8] S. Jaffard, Pointwise smoothness, two-microlocalisation and wavelet coefficients, Pub. Math., 35 (1991), 155-168.

[9] S. Jaffard and Y. Meyer, Wavelet Methods for Pointwise Regularity and Local Oscillations of Functions, Memoirs of the AMS, 123, 1996.

[10] H. Kempka, Local characterization of generalized 2-microlocal spaces, Jenaer Schriften zur Math. \& Inf., 20/06, 2006.

[11] G. Kyriazis, Decomposition systems for function spaces, Studia Math., 157 (2003), 133-169.

[12] J.V. Lévy and S. Seuret, The 2-microlocal formalism, Fractal Geometry and Applications: A Jubilee of Benoit Mandelbrot, Proc. Symp. Pure Math., AMS, Providence RI, vol. 72 (2), 2004.

[13] Y. Meyer and R. Coifman, Wavelets; Calderón-Zygmund and Multilinear Operators, Cambridge Studies in Advanced Math., 48, 1997.

[14] Y. Meyer, Wavelets, Vibrations and Scalings, CRM Monograph Series, AMS, 9, 1997. 
[15] Y. Meyer and H. Xu, Wavelet analysis and chirps, Appl. and Computational Harmonic Analysis, 4 (1997), 366-379.

[16] S. Moritoh and T. Yamada, Two-microlocal Besov spaces and wavelets, Rev. Mat. Iberoamericana, 20 (2004), 277-283.

[17] H.J. Schmeißer and H. Triebel, Topics in Fourier Analysis and Function Spaces, Akademische Verlagsgesellschaft Geest \& Portig, 1987.

[18] H. Triebel, Theory of Function Spaces, Akademische Verlagsgesellschaft Geest \& Portig, 1983.

[19] H. Triebel, A note on wavelet bases in function spaces, Orlicz Centenary Vol., Banach Center Publications, 64 (2004), 193-206.

[20] H. Triebel, Function Spaces and Wavelets on Domains, European Math. Soc. Publishing House, 2008.

[21] H. Triebel, Theory of Function Spaces III, Birkhäuser, 2006.

[22] P. Wojtaszczyk, A Mathematical Introduction to Wavelets, Cambridge University Press, London Math. Society Student Texts, 37, 1997.

[23] H. Hong, Généralisation de la théorie des chirps à divers cadres fonctionnels et application à leur analyse par ondelettes, $\mathrm{Ph}$. D. thesis, Université Paris IX Dauphine, 1996.

Mathematical Institute

Friedrich Schiller University

Ernst-Abbe-Platz 2

07737 Jena

Germany

(E-mail : henning.kempa@uni-jena.de) 


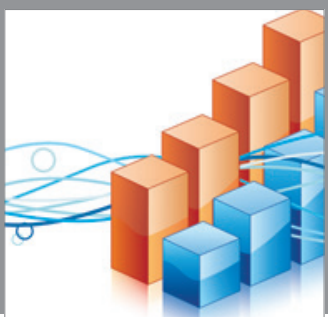

Advances in

Operations Research

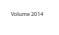

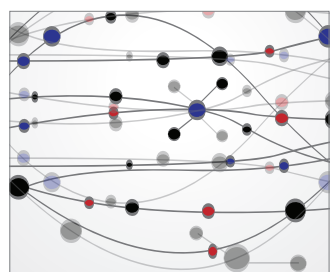

\section{The Scientific} World Journal
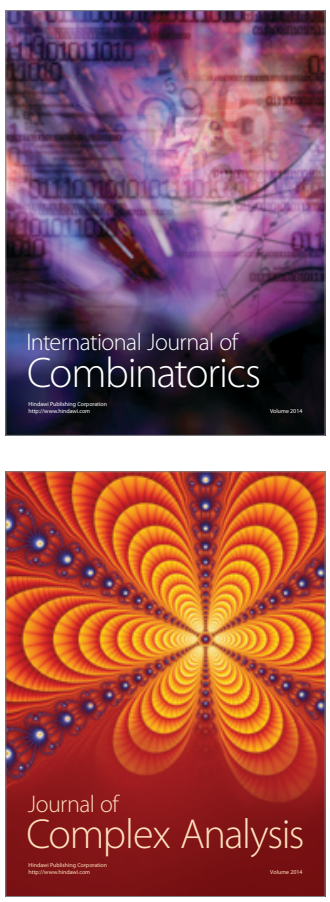

International Journal of

Mathematics and

Mathematical

Sciences
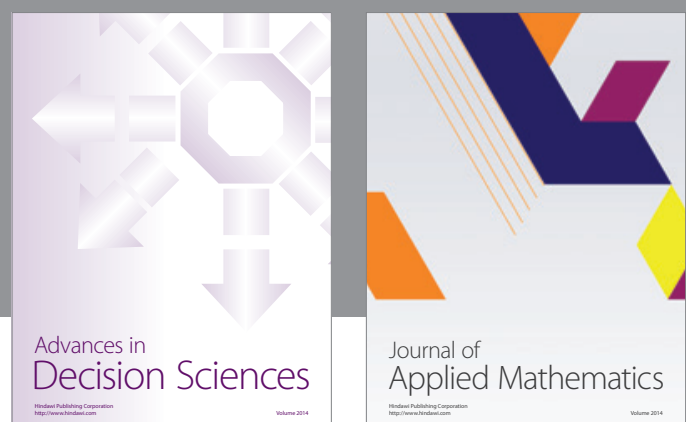

Journal of

Applied Mathematics
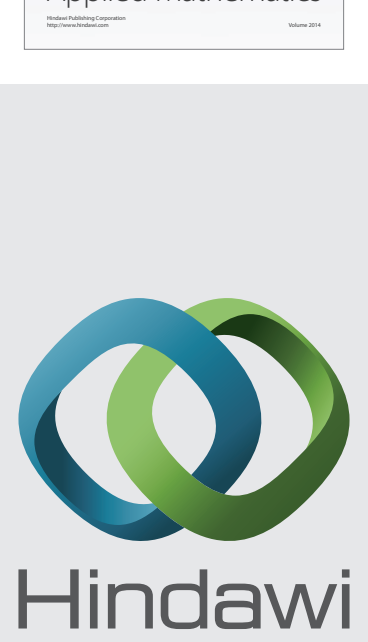

Submit your manuscripts at http://www.hindawi.com
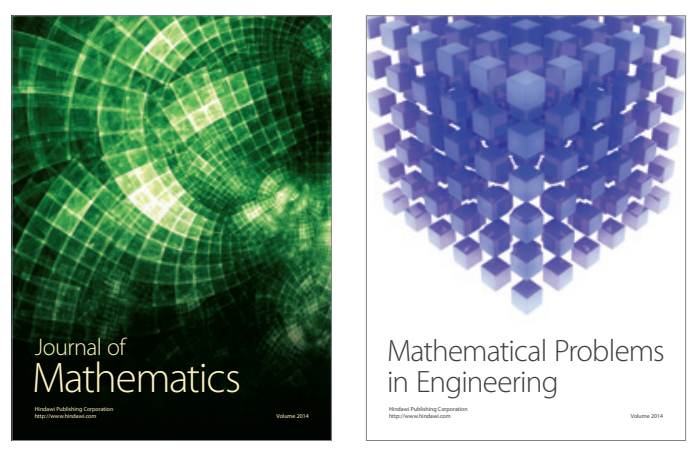

Mathematical Problems in Engineering
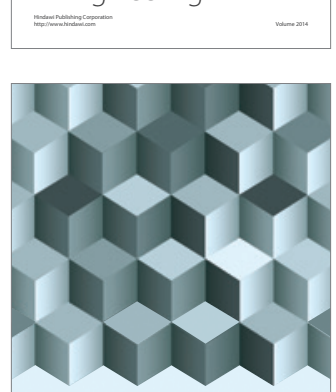

Journal of

Function Spaces
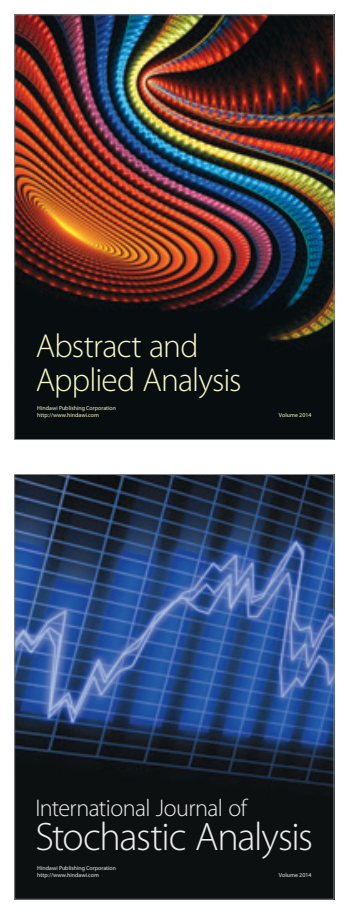

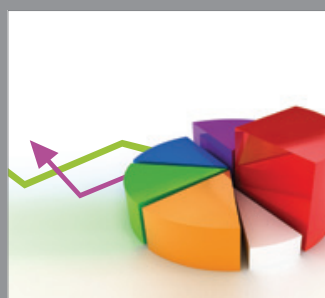

ournal of

Probability and Statistics

Promensencen
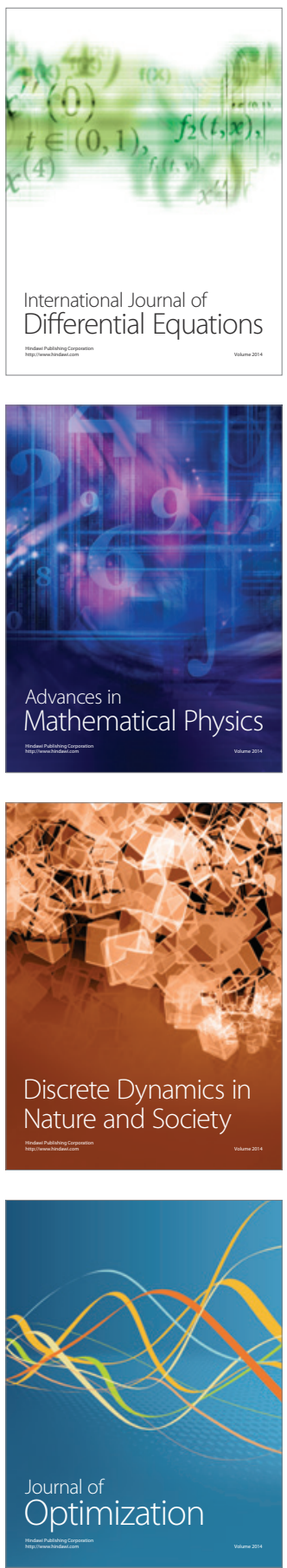